Document downloaded from:

http://hdl.handle.net/10251/156951

This paper must be cited as:

Rufino Marins, TR.; Dos Anjos, AA.; Rodrigo Peñarrocha, VM.; Rubio Arjona, L.; Reig, J.; Amaral De Souza, RA.; Yacoub, MD. (2019). Fading Evaluation in the mm-Wave Band. IEEE Transactions on Communications. 67(12):8725-8738. https://doi.org/10.1109/TCOMM.2019.2941493

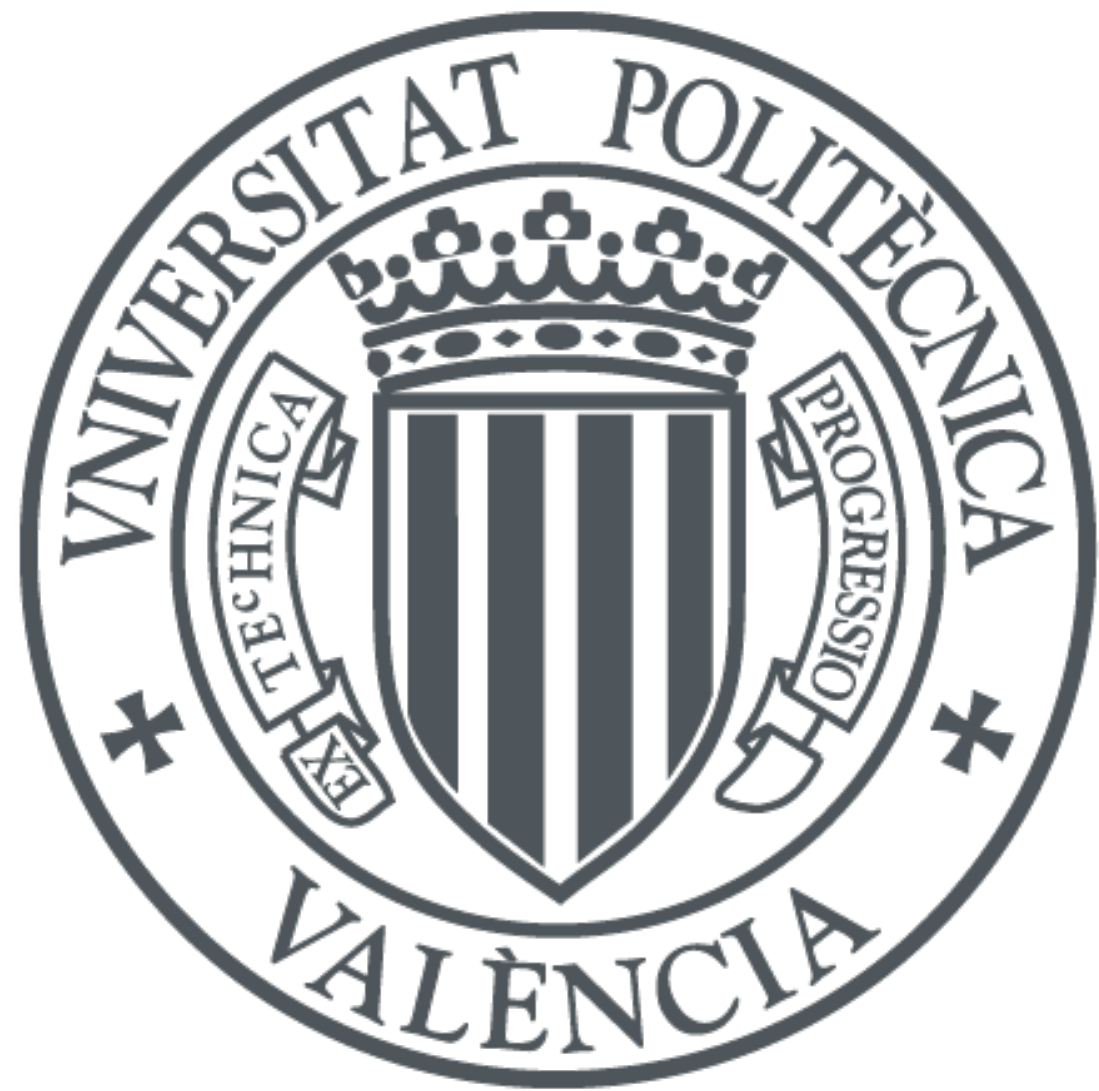

The final publication is available at

https://doi.org/10.1109/TCOMM.2019.2941493

Copyright Institute of Electrical and Electronics Engineers

Additional Information 


\title{
Fading Evaluation in the mm-wave Band
}

\author{
Tiago R. R. Marins, André A. dos Anjos, Vicent Miquel Rodrigo Peñarrocha, \\ Lorenzo Rubio, Senior Member, IEEE, Juan Reig, Senior Member, IEEE, Rausley
}

A. A. de Souza, Member, IEEE and Michel Daoud Yacoub, Member, IEEE

\begin{abstract}
A thorough millimeter-wave measurement campaign is carried out in an indoor environment with an aim at characterizing the short-term fading channel behavior. The measurements are conducted in a variety of scenarios, with frequencies ranging from $55 \mathrm{GHz}$ to $65 \mathrm{GHz}$, in line-of-sight and nonline-of-sight conditions, and combinations of horizontal and vertical polarizations at both transmitter and receiver. A number of fading models are tested, namely Rayleigh, Rice, Nakagami- $m, \alpha-\mu, \kappa-\mu$, $\eta-\mu$, and $\alpha-\eta-\kappa-\mu$. The statistics under analysis are those characterizing the fading amplitude and the frequency selectivity. In particular, the probability density and cumulative distribution functions for the former and level crossing rate per bandwidth unit for the latter are the respective first- and second-order statistics used. To this end, from the experimental data, the parameters of the models are estimated and the corresponding theoretical curves are plotted and compared with the empirical ones. Whereas the required theoretical formulations of the first-order statistics of these models are already well known, those of the second-order statistics as well as these fitting process in such a band shown here are unprecedented in the literature.
\end{abstract}

This work was supported in part by CNPq under Grant 304248/2014-2 and Grant 308365/2017-8 and in part by RNP, with resources from MCTIC, Grant No. No 01250.075413/2018-04, under the Radiocommunication Reference Center (Centro de Referência em Radiocomunicações - CRR) project of the National Institute of Telecommunications (Instituto Nacional de Telecomunicações - Inatel), Brazil, and by the Ministerio de Economía, Industria y Competitividad of the Spanish Government under the national project TEC2017-86779-C2-2-R, through the Agencia Estatal de Investigación (AEI) and the Fondo Europeo de Desarrollo Regional (FEDER).

Tiago R. R. Marins, André A. dos Anjos, and R. A. A. de Souza are with National Institute of Telecommunications - INATEL, Santa Rita do Sapucaí - MG - Brazil (Tel:+55 (35) 3471 9200), e-mail: \{tiagomarins; andre-anjos; rausley\}@inatel.br).

M. D. Yacoub is with the Wireless Technology Laboratory (WissTek), Department of Communications, School of Electrical and Computation Engineering, State University of Campinas, DECOM/FEEC/UNICAMP, 13083-852 Campinas, SP, Brazil (michel@decom.fee.unicamp.br).

Vicent Miquel Rodrigo Peñarrocha, Lorenzo Rubio and Juan Reig are with the Telecommunications and Multimedia Applications Research Institute (iTEAM) at the Universitat Politècnica de València, Camino de Vera, s/n. 46022 Valencia, Spain (email: \{vrodrigo; lrubio; jreigp\}@dcom.upv.es). 


\section{Index Terms}

Millimeter wave communication, small-scale fading, measurement campaign, statistical analysis, frequency domain level crossing rate.

\section{INTRODUCTION}

Advanced wireless communications systems, e.g. the fifth generation (5G) and beyond, shall operate in the millimeter-wave (mm-wave) band in order to circumvent the scarcity of frequency band in the lower portion of the spectrum [1]-[3]. The mm-wave band - spanning from near 30 to $300 \mathrm{GHz}$ - is still virtually unexplored, providing an abundance of resources able to accommodate the multi-Gbps data rates of the new wireless traffic services, a requirement impossible to be met in the sub-6 GHz. The spectrum availability in the mm-wave band is indeed an irrefutable attraction, but the propagation conditions in it are still a matter of investigation.

Several measurement campaigns have been carried out to model the propagation channel in mm-wave frequencies [4]-[9]. It is observed, however, that a considerable effort has been steered towards investigating the large-scale path loss behavior [10]-[12]. In contrast, in spite of its importance, little attention has been paid to the corresponding statistics of the smallscale fading, caused by multipath propagation [13].

Interestingly, many of the stochastic channel models for mm-wave communication systems available in literature assume Rayleigh or Rice distributions for the small-scale fading amplitudes in non-line-of-sight (nLoS) and line-of-sight (LoS) scenarios, respectively [7], [8], [13]-[15]. In [14], the short-term fading amplitude for LoS measurements taken in three corridors of an office block at frequency $60 \mathrm{GHz}$ with a bandwidth of $1 \mathrm{GHz}$ was modeled as a Rician distribution. Values of mean and standard deviation of the Rice factor have been reported for two antenna types, i.e. an open-ended waveguide and lens. Other interesting results have been shown in [13], where the small-scale fading statistics obtained from a $28 \mathrm{GHz}$ outdoor measurement campaign revealed that the Rice density was more suitable than Rayleigh even in nLoS conditions. A glance at some of the published works for different mm-wave scenarios e.g. [13], [15], [16], shows that more elaborate fading models must be used so that the true behavior of the fading channel can be better captured.

The diversity of scenarios envisaged for the mm-wave band will certainly lead to a variety of propagation conditions, which are less likely to be accommodated by the classical fading models. Rayleigh, Hoyt, Rice, Nakagami- $m$ and Weibull will certainly apply, but they may lack 
flexibility to accommodate more intricate situations. Some flexible models, such as $\alpha-\mu, \kappa-\mu$, and $\eta-\mu$ may serve this purpose, but, again, perhaps more generality may be required. Some researches have already assessed the use of multiparameter distributions to model field data in mm-wave frequencies [16]-[18]. However, the results obtained in one or another case have not reproduced some peculiar effect of the behavior of the signal in mm-wave band [16].

Recently, a very general and unifying, physically-based, complex fading model, namely $\alpha$ $\eta-\kappa-\mu$, has been proposed [19]. It accounts for virtually all relevant short-term propagation phenomena, including the following: nonlinearity of the medium, power of the scattered waves, power of the dominant components, and multipath clustering. The model has been described by means of its envelope and phase probability density functions (sPDFs). Due to its multiparameter characteristic, this distribution is able to accommodate an enormous number of fading scenarios. The said model comprises all of the most relevant complex-based fading scenarios found in the literature. Additionally, it can be used to better fit some specific region of the density, e.g. the lower tail region. Apart from this characteristic, a distinguished feature of this distribution is its inherent capability to reproduce multimodality [19]. A due discussion, however, concerns the need for such a general model in light of the difficulty in applying it. Some brief reflections on these issues follow next. Undoubtedly, any common theory attempting to unify several scattered models giving rise to new ones per se is always an interesting topic to pursue. The counterpart of this is that such an unifying theory always leads to mathematical formulations usually more intricate than those comprised by it. And the $\alpha-\eta-\kappa-\mu$ fading model is no exception. Of course, even if only for theoretical purposes, a general fading model built upon physical phenomena may be of interest. But there is much more than a theoretical interest in investigating the application of this model. The field of mm-wave is still barely explored, so that the interactions of the electromagnetic waves and the environment remain to be better known. A fundamental question to be answered is: are the available fading models able to accommodate the possible different situations arising in this new scenario? In other words, is there a need for new models? Note that at these higher frequencies, the irregularities of the surfaces are already on the order of magnitude of the wavelength. Therefore, these surfaces are perceived as rougher, resulting in higher diffusion. Thus, the partial waves of the resulting scattering process may present phase correlation due to the spatially correlated surfaces. Such a correlation may be modeled through different forms of imbalance of the in-phase and quadrature components of the fading process, and this is largely and flexibly available in the $\alpha-\eta-\kappa-\mu$ fading model. (The reader 
is referred to the Section "Conclusions and Challenge" of [19].) Because of its newness as well as comprehensiveness, several issues remain to be investigated, thus creating an enormous opportunity for future researches. Very recently in [20] higher order statistics for the $\alpha-\eta-\kappa-\mu$ model have been derived, namely level crossing rate $\left(\mathrm{LCR}_{\mathrm{t}}\right)$, average fade duration $\left(\mathrm{AFD}_{\mathrm{t}}\right)$, and phase crossing rate $\left(\mathrm{PCR}_{\mathrm{t}}\right)^{1}$.

In addition to the variability of the signal amplitude as a function of time or distance, multipath is also known to provoke frequency selectivity. In this sense, finding the PDF that yields the best fit for the fading amplitude is as relevant as knowing how the amplitude varies as a function of the frequency, a phenomenon that may be captured by the frequency domain level crossing rate $\left(\mathrm{LCR}_{\mathrm{f}}\right)[21]$.

In this paper, a thorough mm-wave measurement campaign is carried out in an indoor environment aiming at characterizing the short-term fading channel behavior. The chosen frequencies cover the full range from $55 \mathrm{GHz}$ to $65 \mathrm{GHz}$ band, in LoS and nLoS conditions, with combinations of horizontal and vertical polarizations at both transmitter and receiver. In addition to these thorough practical measurements, the following are contributions appearing in the paper.

1) Selection of the fading model PDF, chosen among Rayleigh, Rice, Nakagami- $m, \alpha-\mu, \kappa-\mu$, $\eta-\mu$, and $\alpha-\eta-\kappa-\mu$ that best fits the experimental data using the following goodness-of-fit (GoF) metrics: normalized mean square error (NMSE), Kolmogorov-Smirnov (KS), and Akaike information criterion (AIC).

2) Derivation of an exact closed-form expression for the $\mathrm{LCR}_{\mathrm{f}}$ of the $\alpha$ - $\mu$ fading model.

3) Derivation of an exact closed-form expression for the $\mathrm{LCR}_{\mathrm{f}}$ of the $\kappa$ - $\mu$ fading model.

4) Derivation of an exact $\mathrm{LCR}_{\mathrm{f}}$ expression for the $\eta$ - $\mu$ fading model in terms of a single proper integral.

5) Derivation of an exact $\mathrm{LCR}_{\mathrm{f}}$ expression for the $\alpha-\eta-\kappa-\mu$ fading model in terms of a single proper integral.

6) Selection of the fading model $\mathrm{LCR}_{\mathrm{f}}$, chosen among Rayleigh, Rice, Nakagami- $m, \alpha-\mu, \kappa-$ $\mu, \eta-\mu$, and $\alpha-\eta-\kappa-\mu$ that best fits the experimental data using the following GoF metrics: NMSE and AIC.

${ }^{1}$ The acronyms for level crossing rate, average fade duration, and phase crossing rate are followed by a subscript $t$ to denote that these statistics are obtained having as attribute the time (level crossing per time, time below level, and phase crossing per time). As shall be seen, in this paper, we use $\mathrm{LCR}_{\mathrm{f}}$ to denote level crossing rate per frequency. 
This paper is organized as follows. Section II briefly revisits both the main parameters of the fading models under test and GoF methods used. It also derives the expressions of $\mathrm{LCR}_{\mathrm{f}}$ for some of the main multiparameter distributions. The measurement setup and the measurement environment are described in Section III. Section IV analyzes the numerical results obtained from the measured data. Finally, Section V draws some conclusions.

\section{Preliminaries}

\section{A. Fading Models Under Test}

The fading distributions to be tested here include the following: (i) the best-known models, namely, Rayleigh, Rice, Nakagami-m; (ii) the already popular general models, namely $\alpha-\mu, \eta-\mu$, and $\kappa-\mu$; and (iii) the very recently and most general model, namely $\alpha-\eta-\kappa-\mu$. Because of its newness, and because it comprises all of the other ones, it is certainly of interest to revisit the definition of the parameters involved in $\alpha-\eta-\kappa-\mu$. The $\alpha-\eta-\kappa-\mu$ envelope $R$ can be written as [19, Eqn. (9)]

$$
R^{\alpha}=\sum_{i=1}^{\mu_{x}}\left(X_{i}+\lambda_{x_{i}}\right)^{2}+\sum_{i=1}^{\mu_{y}}\left(Y_{i}+\lambda_{y_{i}}\right)^{2}
$$

in which: (i) $\alpha>0$ quantifies the nonlinearity of the medium; (ii) $X_{i}$ and $Y_{i}$ are mutually independent Gaussian processes; (iii) $\mathbb{E}\left(X_{i}\right)=\mathbb{E}\left(Y_{i}\right)=0$, with $\mathbb{E}(\cdot)$ denoting the expectation operator; (iv) $\mathbb{E}\left(X_{i}^{2}\right)=\sigma_{x}^{2} ; \mathbb{E}\left(Y_{i}^{2}\right)=\sigma_{y}^{2}$; (v) $\lambda_{x_{i}}$ and $\lambda_{y_{i}}$ are, respectively, the mean values of the in-phase and quadrature components of the multipath waves of cluster $i$; (v) $\mu_{x}$ and $\mu_{y}$ are the number of multipath clusters of in-phase and quadrature components, respectively. The multiparameter PDF of the $\alpha-\eta-\kappa-\mu$ envelope model is presented in three parametrization formats, namely Raw [19, Eqn. (17)], Local [19, Eqn. (23)], and Global Parametrization [19, Eqn. (29)]. For convenience, i.e. because this is the one used here, only the Global Parametrization is described. In the Global Parametrization, the following are the parameters (all positive): (i) $\alpha$ denotes the nonlinearity of the medium; (ii) $\eta=\mu_{x} \sigma_{x}^{2} /\left(\mu_{y} \sigma_{y}^{2}\right)$ defines the ratio of the total power of the in-phase and quadrature scattered waves of the multipath clusters; (iii) $\kappa=\left(\lambda_{x}^{2}+\lambda_{y}^{2}\right) /\left(\mu_{x} \sigma_{x}^{2}+\mu_{y} \sigma_{y}^{2}\right)$ gives the ratio of the total power of the dominant components and the total power of scattered waves; (iv) $\mu=\left(\mu_{x}+\mu_{y}\right) / 2$ concerns the number of multipath clusters; (v) $p=\mu_{x} / \mu_{y}$ depicts the ratio of the number of multipath clusters of in-phase and quadrature signals; (vi) $q=\lambda_{x}^{2} \mu_{y} \sigma_{y}^{2} /\left(\lambda_{y}^{2} \mu_{x} \sigma_{x}^{2}\right)$ describes the ratio of two ratios: the ratio of the power of the dominant components to the power of the scattered waves of the in-phase signal 
and its counterpart for the quadrature signal; and (vii) $\hat{r}^{\alpha}=\mathbb{E}\left(R^{\alpha}\right)=\mu_{x} \sigma_{x}^{2}+\lambda_{x}^{2}+\mu_{y} \sigma_{y}^{2}+\lambda_{y}^{2}$. The number of distributions obtainable from the $\alpha-\eta-\kappa-\mu$ model as particular cases is enormous and is far from having been explored in its entirety yet. A detailed explanation for the known special cases and also the meaning of each parameter can be found in [19]. The PDFs of normalized envelope $\rho=R / \sqrt[\alpha]{\mathbb{E}\left(R^{\alpha}\right)}$ of the target fading models to be tested here are given as follows: ${ }^{2}$

1) Rayleigh

$$
f_{P}(\rho)=2 \rho \exp \left(-\rho^{2}\right)
$$

2) Rice (with parameter $\kappa$ )

$$
f_{P}(\rho)=2(\kappa+1) \rho \exp \left(-\kappa-(\kappa+1) \rho^{2}\right) I_{0}(2 \sqrt{\kappa(1+\kappa)} \rho)
$$

3) Nakagami- $m$ (with parameter $\mu$ )

$$
f_{P}(\rho)=\frac{2 \mu^{\mu} \rho^{2 \mu-1} \exp \left(-\mu \rho^{2}\right)}{\Gamma(\mu)}
$$

4) $\alpha-\mu$

$$
f_{P}(\rho)=\frac{\alpha \mu^{\mu} \rho^{\alpha \mu-1}}{\Gamma(\mu) \exp \left(\mu \rho^{\alpha}\right)}
$$

5) $\kappa-\mu$

$$
f_{P}(\rho)=\frac{2 \mu(1+\kappa)^{\frac{\mu+1}{2}}}{\kappa^{\frac{\mu-1}{2}} \exp (\mu \kappa)} \rho^{\mu} \exp \left(-\mu(1+\kappa) \rho^{2}\right) I_{\mu-1}(2 \mu \sqrt{\kappa(1+\kappa)} \rho)
$$

6) $\eta-\mu$

$$
f_{P}(\rho)=\frac{2 \sqrt{\pi}(1+\eta)^{\mu+\frac{1}{2}} \mu^{\mu+\frac{1}{2}} \rho^{2 \mu}}{\sqrt{\eta}(1-\eta)^{\mu-\frac{1}{2}} \Gamma(\mu)} \exp \left(-\frac{\mu(1+\eta)^{2} \rho^{2}}{2 \eta}\right) I_{\mu-\frac{1}{2}}\left(\frac{\mu(1-\eta)^{2} \rho^{2}}{2 \eta}\right)
$$

7) Finally, $\alpha-\eta-\kappa-\mu^{3}$

$$
f_{P}(\rho)=\int_{0}^{2 \pi} f_{P, \Theta}(\rho, \theta) d \theta
$$

${ }^{2}$ For homogeneity, the parameters of all distributions are given in terms of those of the more general one.

${ }^{3}$ Other functional forms for the PDF of the $\alpha-\eta-\kappa-\mu$ envelope can be found in [19]. 
where $f_{P, \Theta}(\rho, \theta)$ is given by

$$
\begin{aligned}
f_{P, \Theta}(\rho, \theta) & =\frac{\alpha \mu^{2} p(\eta+1)^{2}(\kappa+1)^{\frac{\mu}{2}+1} \rho^{\frac{\alpha}{2}(\mu+2)-1}|\sin (\theta)|^{\frac{\mu}{p+1}}|\cos (\theta)|^{\frac{\mu p}{p+1}}}{2 \eta(p+1)^{2}\left(\frac{\kappa}{\eta q+1}\right)^{\frac{\mu}{2}-1}(\eta q)^{\frac{\mu p}{2(p+1)}-\frac{1}{2}} \exp \left(\frac{\kappa \mu(\eta+1)(q p+1)}{(p+1)(\eta q+1)}\right)} \\
& \times \exp \left(-\frac{\mu(\eta+1)(\kappa+1)\left(\eta \sin ^{2}(\theta)+p \cos ^{2}(\theta)\right)}{\eta(p+1)} \rho^{\alpha}\right) \operatorname{sech}\left(\frac{2 \mu p(\eta+1) \cos (\theta)}{\eta(p+1)} \sqrt{\frac{\eta \kappa q(\kappa+1)}{\eta q+1}} \rho^{\frac{\alpha}{2}}\right) \\
& \times \exp \left(\frac{2 \mu(\eta+1) \cos (\theta-\phi)}{\eta(p+1)} \sqrt{\frac{\eta \kappa(\kappa+1)\left(\eta+q p^{2}\right)}{\eta q+1}} \rho^{\frac{\alpha}{2}}\right) \operatorname{sech}\left(\frac{2 \mu(\eta+1) \sin (\theta)}{p+1} \sqrt{\frac{\kappa(\kappa+1)}{\eta q+1}} \rho^{\frac{\alpha}{2}}\right) \\
& \times I_{\frac{\mu}{p+1}}-1 \\
& \left(\frac{2 \mu(\eta+1)|\sin (\theta)|}{p+1} \sqrt{\frac{\kappa(\kappa+1)}{\eta q+1}} \rho^{\frac{\alpha}{2}}\right) I_{\frac{\mu p}{p+1}-1}\left(\frac{2 \mu p(\eta+1)|\cos (\theta)|}{\eta(p+1)} \sqrt{\frac{\eta \kappa q(\kappa+1)}{\eta q+1}} \rho^{\frac{\alpha}{2}}\right)
\end{aligned}
$$

\section{B. New LCR Expressions}

The behavior of the wireless communications channel is drastically affected by the multipath propagation phenomenon. In addition to provoking a random fluctuation of the signal as a function of time (or distance), multipath also causes frequency selectivity. Characterizing such statistics is certainly of interest.

The high demand for data rates is leading systems to use larger bandwidth, often with tens to hundreds of $\mathrm{MHz}$, or even several $\mathrm{GHz}$, rendering the dispersion of channels (frequency selectivity) a major issue in the design of air interfaces for digital communications. This can directly affect the correlation or orthogonality between the subcarriers in multi-carrier systems such as orthogonal frequency division multiplexing (OFDM), commonly used in broadcast and mobile communications [22]. In such systems, many of the methods developed for narrow-band, single-carrier transmission can be employed in the frequency-domain. One of the metrics used to assess the variability of the amplitude as a function of the frequency is the so-called LCR $_{\mathrm{f}}$.

In this section, the $\mathrm{LCR}_{\mathrm{f}}$ expressions for the $\alpha-\mu, \kappa-\mu, \eta-\mu$, and $\alpha-\eta-\kappa-\mu$ fading models are shown. These expressions are then used to fit field data measurements carried out in the $55 \mathrm{GHz}$ to $65 \mathrm{GHz}$ band in Section IV. To the best of the authors' knowledge, both the expressions as well as their fitting to field data in such a band are unprecedented in the literature.

The $\mathrm{LCR}_{\mathrm{f}}$ refers to the average number of times the signal crosses a given amplitude level in the positive (or negative) direction per bandwidth unit. In [21], the $\mathrm{LCR}_{\mathrm{f}}$ has been derived for the Nakagami- $m$ distribution. Here, we follow the same approach to obtain the $\mathrm{LCR}_{\mathrm{f}}$ for 
all mentioned fading models. As a matter of fact, in essence, the approach used in [21] is the same as that developed in [23] and then followed for the other fading environments [21], [24] but having frequency, rather than time, as the crossing attribute. Interestingly, using the due procedure, but having frequency rather than time as the crossing attribute, yields exactly the same functional forms as those of [20], [23], [25]-[29]. Hence, the $\operatorname{LCR}_{\mathrm{f}}$ statistics, $N_{R_{\mathrm{f}}}(\rho)$, for the various environments, in which $\rho$ is the amplitude level normalized to the its rms value and $\ddot{\psi}(0)$ is the second derivative with respect to frequency of the frequency autocorrelation function at zero, are given as follows ${ }^{4}$ :

1) For Rayleigh

$$
N_{R_{\mathrm{f}}}(\rho)=\frac{\rho \sqrt{-\ddot{\psi}(0)}}{\sqrt{\pi} \exp \left(\rho^{2}\right)} .
$$

2) For Rice (with parameter $\kappa$ )

$$
N_{R_{\mathrm{f}}}(\rho)=\frac{\rho \sqrt{-\ddot{\psi}(0)(\kappa+1)} I_{0}(2 \rho \sqrt{\kappa(\kappa+1)})}{\sqrt{\pi} \exp (\kappa) \exp \left((\kappa+1) \rho^{2}\right)} .
$$

3) For Nakagami-m (with parameter $\mu$ )

$$
N_{R_{\mathrm{f}}}(\rho)=\frac{\rho^{2 \mu-1} \mu^{\mu-\frac{1}{2}} \sqrt{-\ddot{\psi}(0)}}{\sqrt{\pi} \Gamma(\mu) \exp \left(\mu \rho^{2}\right)} .
$$

4) For $\alpha-\mu$

$$
N_{R_{\mathrm{f}}}(\rho)=\frac{\rho^{\alpha\left(\mu-\frac{1}{2}\right)} \mu^{\mu-\frac{1}{2}} \sqrt{-\ddot{\psi}(0)}}{\sqrt{\pi} \Gamma(\mu) \exp \left(\mu \rho^{\alpha}\right)} .
$$

5) For $\kappa-\mu$

$$
N_{R_{\mathrm{f}}}(\rho)=\frac{\rho^{\mu} \sqrt{-\ddot{\psi}(0) \mu(1+\kappa)^{\mu}} I_{\mu-1}(2 \mu \rho \sqrt{\kappa(1+\kappa)})}{\sqrt{\pi \kappa^{(\mu-1)}} \exp (\mu \kappa) \exp \left(\mu(1+\kappa) \rho^{2}\right)} .
$$

${ }^{4}$ It is noteworthy that in [20], [25]-[29], the $\mathrm{LCR}_{\mathrm{t}}$ formulations have been derived for an isotropic environments, in which case $\ddot{\psi}(0)=-2 \pi^{2} f_{\mathrm{d}}^{2}$, with $\ddot{\psi}(0)$ being the second derivative with respect to time of the temporal autocorrelation function at zero and $f_{\mathrm{d}}$ is the maximum Doppler shift. An anisotropic environment can be modeled by using $f_{\mathrm{d}}=\left(-\ddot{\psi}(0) /\left(2 \pi^{2}\right)\right)^{1 / 2}$ in the formulations. Now the $\mathrm{LCR}_{\mathrm{f}}$ is finally expressions are attained bearing in mind that $\ddot{\psi}(0)$ signifies the second derivative with respect to frequency of the frequency autocorrelation function at zero. 
6) For $\eta-\mu$ the $\mathrm{LCR}_{\mathrm{f}}$ can be calculated by

$$
\begin{aligned}
& N_{R_{\mathrm{f}}}(\rho)=\frac{((1+\eta) \mu)^{2 \mu-\frac{1}{2}} \rho^{4 \mu-1} \sqrt{-\ddot{\psi}(0)}}{\sqrt{2 \pi} \eta^{\mu} 2^{2 \mu-2} \Gamma^{2}(\mu)} \times \\
& \quad \int_{0}^{\frac{\pi}{2}} \sin (2 \theta)^{2 \mu-1} \sqrt{1+\eta-(1-\eta) \cos (2 \theta)} \exp \left(-\frac{\left((1+\eta)^{2}+\left(1-\eta^{2}\right) \cos (2 \theta)\right) \mu \rho^{2}}{2 \eta}\right) d \theta
\end{aligned}
$$

7) Finally, for $\alpha-\eta-\kappa-\mu$

$$
N_{R_{\mathrm{f}}}(\rho)=\int_{0}^{2 \pi} N_{R_{\mathrm{f}}}(\rho, \theta) d \theta
$$

where $N_{R_{\mathrm{f}}}(\rho, \theta)$ is given by

$N_{R_{\mathrm{f}}}(\rho, \theta)=$

$$
\begin{aligned}
& \underline{\sqrt{-\ddot{\psi}(0)} \rho^{\frac{1}{2} \alpha(2+\mu)} \frac{\kappa}{1+q \eta+\kappa+q \eta \kappa}\left(\frac{1+p-\mu}{2+2 p}\right) \frac{q \eta \kappa}{1+q \eta+\kappa+q \eta \kappa}\left(\frac{1+p-p \mu}{2+2 p}\right)|\cos (\theta)|^{\left(\frac{p \mu}{1+p}\right)}|\sin (\theta)|^{\left(\frac{\mu}{1+p}\right)}\left(d^{2} \eta \cos (\theta)^{2}+p \sin (\theta)^{2}\right)} \\
& \sqrt{\pi}(1+d)^{4} \eta\left(\frac{(1+p)}{(1+d)^{2}(1+\eta)(1+\kappa) \mu}\right)^{3 / 2} \sqrt{\frac{\rho^{\alpha}\left(d^{2} \eta \cos (\theta)^{2}+p \sin (\theta)^{2}\right)}{p}} \\
& \times \exp \left(\frac{2 \rho^{\alpha / 2}(1+q \eta)(1+\kappa) p \sqrt{\frac{q \eta \kappa}{1+q \eta+\kappa+q \eta \kappa}} \cos (\theta)-\rho^{\alpha}(1+q \eta)(1+\kappa) p \cos (\theta)^{2}}{((1+\eta) \mu)^{-1}(1+p) \eta(1+q \eta)}\right) \\
& \times \exp \left(\frac{\eta\left(2 \rho^{\alpha / 2}(1+q \eta)(1+\kappa) \sqrt{\frac{\kappa}{1+q \eta+\kappa+q \eta \kappa}} \sin (\theta)-(1+p q) \kappa-\rho^{\alpha}(1+q \eta)(1+\kappa) \sin (\theta)^{2}\right)}{((1+\eta) \mu)^{-1}(1+p) \eta(1+q \eta)}\right) \\
& \times I_{\frac{\mu}{1+p}}-1\left(\frac{2 \rho^{\alpha / 2}(1+\eta)(1+\kappa) \mu \sqrt{\frac{\kappa}{1+q \eta+\kappa+q \eta \kappa}}|\sin (\theta)|}{1+p}\right) \operatorname{I\frac {p\mu }{1+p}-1}\left(\frac{2 \rho^{\alpha / 2}(1+\eta)(1+\kappa) \mu p \sqrt{\frac{q \eta \kappa}{1+q \eta+\kappa+q \eta \kappa}}|\cos (\theta)|}{(1+p) \eta}\right) \\
& \times \operatorname{sech}\left(\frac{2 \rho^{\alpha / 2}(1+\eta)(1+\kappa) \mu \sqrt{\frac{\kappa}{1+q \eta+\kappa+q \eta \kappa}} \sin (\theta)}{1+p}\right) \operatorname{sech}\left(\frac{2 \rho^{\alpha / 2}(1+\eta)(1+\kappa) \mu p \sqrt{\frac{q \eta \kappa}{1+q \eta+\kappa+q \eta \kappa}} \cos (\theta)}{(1+p) \eta}\right)
\end{aligned}
$$

and $I_{v}(\cdot)$ is the modified Bessel function of the first kind and order $v$ [30, Eqn. (8.406.5)]. In (17) we define the following: (i) $\ddot{\psi}(0)$, as the mean of the second derivative with respect to frequency of the frequency autocorrelation function at zero; (ii) $d$, as the imbalance of the second derivative with respect to frequency of the frequency autocorrelation function at zero between in-phase and quadrature components. Consequently, (i) $2(-\ddot{\psi}(0))^{1 / 2}=\left(-\ddot{\psi}_{x}(0)\right)^{1 / 2}+\left(-\ddot{\psi}_{y}(0)\right)^{1 / 2}$; (ii) $d=\left(-\ddot{\psi}_{x}(0)\right)^{1 / 2} /\left(-\ddot{\psi}_{y}(0)\right)^{1 / 2}$; (iii) $\left(-\ddot{\psi}_{x}(0)\right)^{1 / 2}=2 d(-\ddot{\psi}(0))^{1 / 2} /(1+d)$; (iv) $\left(-\ddot{\psi}_{y}(0)\right)^{1 / 2}=$ $2(-\ddot{\psi}(0))^{1 / 2} /(1+d)$; 


\section{Goodness-of-fit Tests}

The first step towards defining the distribution that best fits the field data concerns the use of some GoF methods to assess how practical and theoretical distributions compare with each other. Practical distributions are obtained empirically through a histogram built from field data. Theoretical distributions are found with the respective parameters estimated from the same field data. In the literature there are a number of statistical criteria to define the choice between one or another model, each of which with specific characteristics [31]-[36]. However, since

they are statistical models, there is no one single criterion that is universally accepted as the best model. Each criterion uses different analysis methods to pick its choice and this can lead to different results when more than one criterion is used. It is noteworthy that the subject of estimation of the relative quality of statistical models is vast and no criterion is unequivocally and unanimously found to be the best. In the analyses conducted here, three different figures of merit or criteria are used, namely NMSE, KS, and AIC. The NMSE, in logarithmic scale, is used for analyses where the focus is to compare the empirical PDF and the theoretical one in order to quantify the PDFs dissimilarity (mean distance). The nonparametric KS test is performed so that the estimated cumulative distribution function (CDF) and the hypothesized CDF are compared. The AIC is used to compare empirical and theoretical statistic distribution models considering dissimilarity, quantity of samples, and number of parameters of the distribution. In these three statistical tests aforementioned, objective figures of merit are obtained that can be used to evaluate the GoF between empirical and theoretical distributions. In NMSE and AIC tests, the chosen distribution is the one with the lowest value. The KS statistical parameter $D$ represents the maximum absolute difference between theoretical and practical distributions. If the sample comes from a given distribution, then $D$ converges to zero almost surely in the limit as the sample size tends to infinity. The corresponding $p$-value is calculated based on the KS parameter $D$ and it represents the reliability of the test considering a given confidence interval. The best-fit performance is achieved by the distribution presenting the lowest $D$ value and highest $p$-value. Because NMSE, KS, and AIC statistical tests use different approaches, the conclusions drawn from them will not necessarily be the same, that is the chosen distribution in one test may not coincide with the one in the others. In addition, in some scenarios, e.g. for severe fading conditions, it may be necessary to evaluate the fitting at the tail of the distribution. In such a case, none of the above metrics seem adequate. A statistical test that best yields an appropriate 
metric in this case is the modified KS test [37]. As well as in the conventional KS test, such a metric is used to quantify the GoF between the empirical and theoretical CDFs, but now with the CDF plotted in log-scale with the objective of highlighting the fitting in amplitude values closer to zero. As a consequence, the modified KS test emphasizes the fitting analysis where the fading is naturally more severe.

\section{Channel Measurements}

\section{A. Measurement Environment}

The channel measurement campaign was carried out in a laboratory of the iTEAM Research Institute at the Universitat Politècnica de València, Spain. The laboratory environment is characterized by the presence of radiofrequency equipments, metallic cupboards, multiple, desks and work tables and benches equipped with several computers and electronic devices and chairs, among other items. The presence of numerous interacting objects, many of them metallic, makes this a dense multipath environment due to different reflection, diffraction, and scattering propagation mechanisms. The laboratory is integrated into a modern building construction, where ceiling and floor are built of reinforced concrete over steel plates with wood and plasterboardspaneled walls. The propagation environment dimensions are $13.5 \times 7 \mathrm{~m}^{2}$ with a height of $2.6 \mathrm{~m}$.

\section{B. Measurement Setup}

The complex channel transfer function $(\mathrm{CTF})$ was measured in the frequency domain using a channel sounder based on a vector network analyzer (VNA), the Keysight N5227A, with a dynamic range of $119 \mathrm{~dB}$ and a maximum output power equal to $+10 \mathrm{dBm}$ from $50 \mathrm{GHz}$ to $67 \mathrm{GHz}$. Q-par wideband antennas with vertical polarization and omnidirectional radiation pattern in azimuth (horizontal plane) were used at the transmitter (Tx) and the receiver $(\mathrm{Rx})$ sides. In addition, three HXI-HLNA low noise amplifiers (LNAs), one at the Tx side and two at the Rx side, phase-stable and very low attenuation cables were used. The Tx antenna was mounted on a tripod at a height of $1.44 \mathrm{~m}$ above the floor. The Rx antenna was placed over a XY linear positioning system emulating a $N \times M$ uniform rectangular array (URA) with an inter-element separation equal to $2 \mathrm{~mm}$ (equivalent to $0.4 \lambda$ at $60 \mathrm{GHz}$ ). The height of the $\mathrm{Rx}$ antenna was also $1.44 \mathrm{~m}$ with respect to the floor. The choice for the $\mathrm{Tx}$ and $\mathrm{Rx}$ height of $1.44 \mathrm{~m}$ is justified as follows. This height is similar to the average height where many obstacles are located in this environment, e.g. central process unit (CPU) boxes, monitors, and different 
TABLE I: Measurement System Parameters

\begin{tabular}{l|c}
\hline \hline Parameter & Value \\
\hline VNA output power & $0 \mathrm{dBm}$ \\
VNA center frequency & $60 \mathrm{GHz}$ \\
VNA SPAN (Bandwidth) & $10 \mathrm{GHz}$ \\
VNA IF Bandwidth & $100 \mathrm{~Hz}$ \\
Frequency points per trace & 2048 \\
Average antenna gain & $5 \mathrm{dBi}$ \\
Average amplifiers gain & $25 \mathrm{~dB}$ \\
Tx antenna height & $1.44 \mathrm{~m}$ \\
Rx antenna height & $1.44 \mathrm{~m}$ \\
Total cables length & $5.5 \mathrm{~m}$ \\
Cable attenuation at $65 \mathrm{GHz}$ & $6 \mathrm{~dB} / \mathrm{m}$ \\
\hline
\end{tabular}

laboratory devices. Therefore, at this height, we may obtain a rich scattering environment with a large number of multipath signals. In the same way, this corresponds to an approximate height of a smartphone handled by its user. Thus, this scenario could closely emulate the one in which cell phones are used. The Tx subsystem was connected to the Port-1 of the VNA and the Rx subsystem was connected to the Port-2. The VNA and the XY positioning system were controlled by a personal computer, measuring the $S_{21}(f)$ scattering parameter, equivalent to the CTF of the propagation channel. Equipment calibration was performed before the measurements procedure to compensate for the attenuation and for any imperfection of the channel sounder components, with the exception of the antennas. Thus, the measured $S_{21}(f)$ parameter includes the channel and the Tx and Rx antennas. An overview of the propagation channel measurement setup can be seen in Fig. 1. For each position of the Rx antenna in the URA, the $S_{21}(f)$ parameter was measured directly from 55 to $65 \mathrm{GHz}$ (a SPAN of $10 \mathrm{GHz}$ in the VNA) using 2048 frequency points, with a frequency resolution about $4.88 \mathrm{MHz}$. The intermediate frequency (IF) filter bandwidth was selected to $100 \mathrm{~Hz}$ to reduce the power level of the noise floor. Table I summarizes the measurement system parameters.

Two different locations for the Tx were considered. In the first location, the Tx-Rx distance was $3.29 \mathrm{~m}$, and a $35 \times 35$ URA in the Rx side was implemented. Three different propagation conditions were investigated:

- Horizontal $(\mathrm{H})$ polarization at the Tx antenna and vertical (V) polarization at the Rx antenna, 


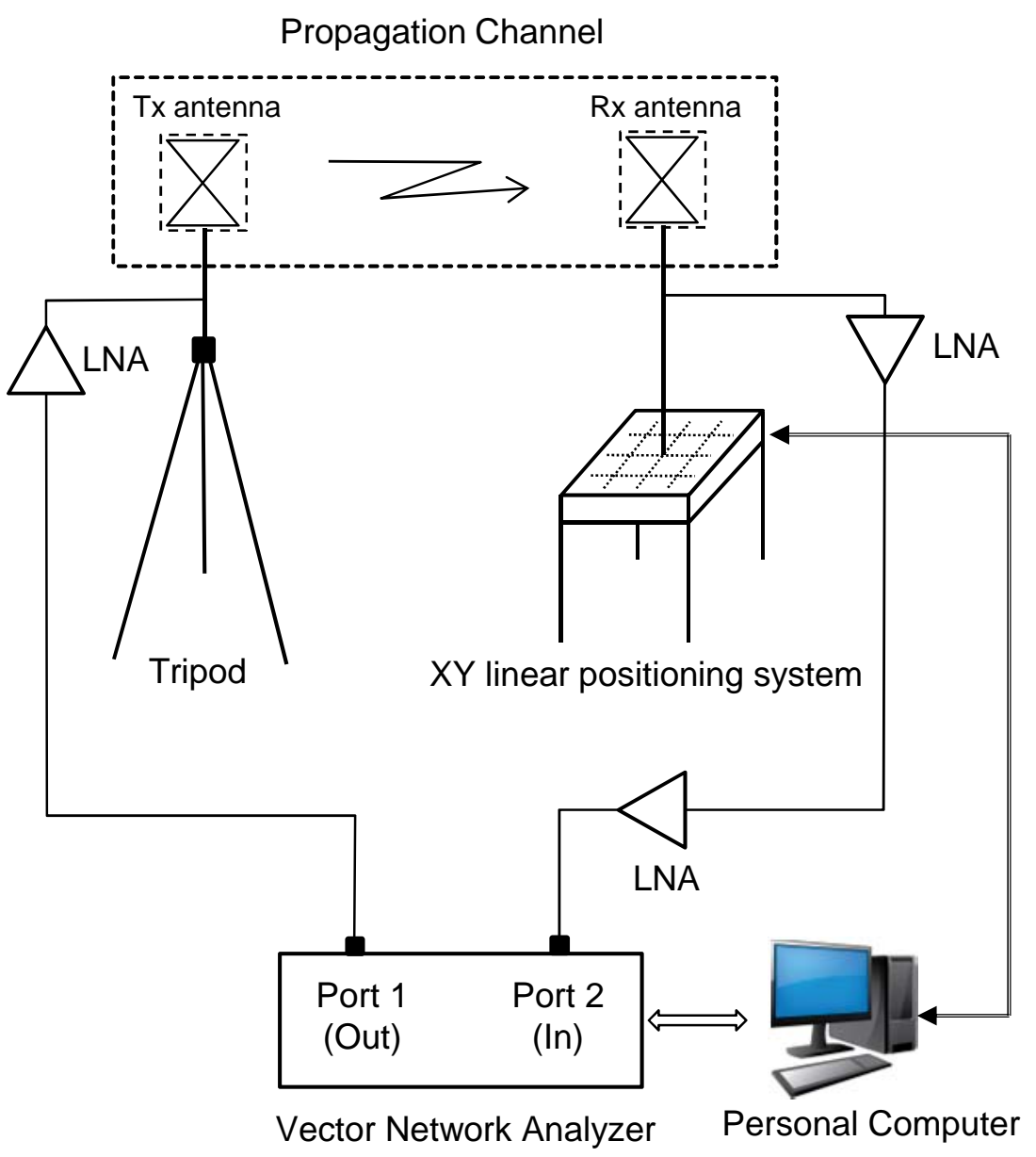

Fig. 1: Overview of the frequency domain propagation channel measurement setup.

i.e., HV polarization, and $\operatorname{LoS}$ conditions, referred to as Scenario 1.

- VV polarization and LoS conditions, referred to as Scenario 2.

- VV polarization and nLoS conditions, where the LoS component was obstructed using radiation-absorbent material as can be seen in Fig. 2. This propagation condition is referred to as Scenario 3.

In the second location, the Tx-Rx distance was $2.77 \mathrm{~m}$, and a 50×50 URA was implemented. In this case, VV polarization and LoS conditions were used. This propagation condition is denoted as Scenario 4. Table II summarizes the characteristics of the defined scenarios.

According to the VNA configuration, the required time to measure the $S_{21}(f)$ scattering parameter for each position of the Rx antenna in the URA was about $36.32 \mathrm{~s}$. Thus the acquisition time of the measurements in Scenarios 1, 2 and 3 was about $12.3 \mathrm{~h}$, whereas in the Scenario 

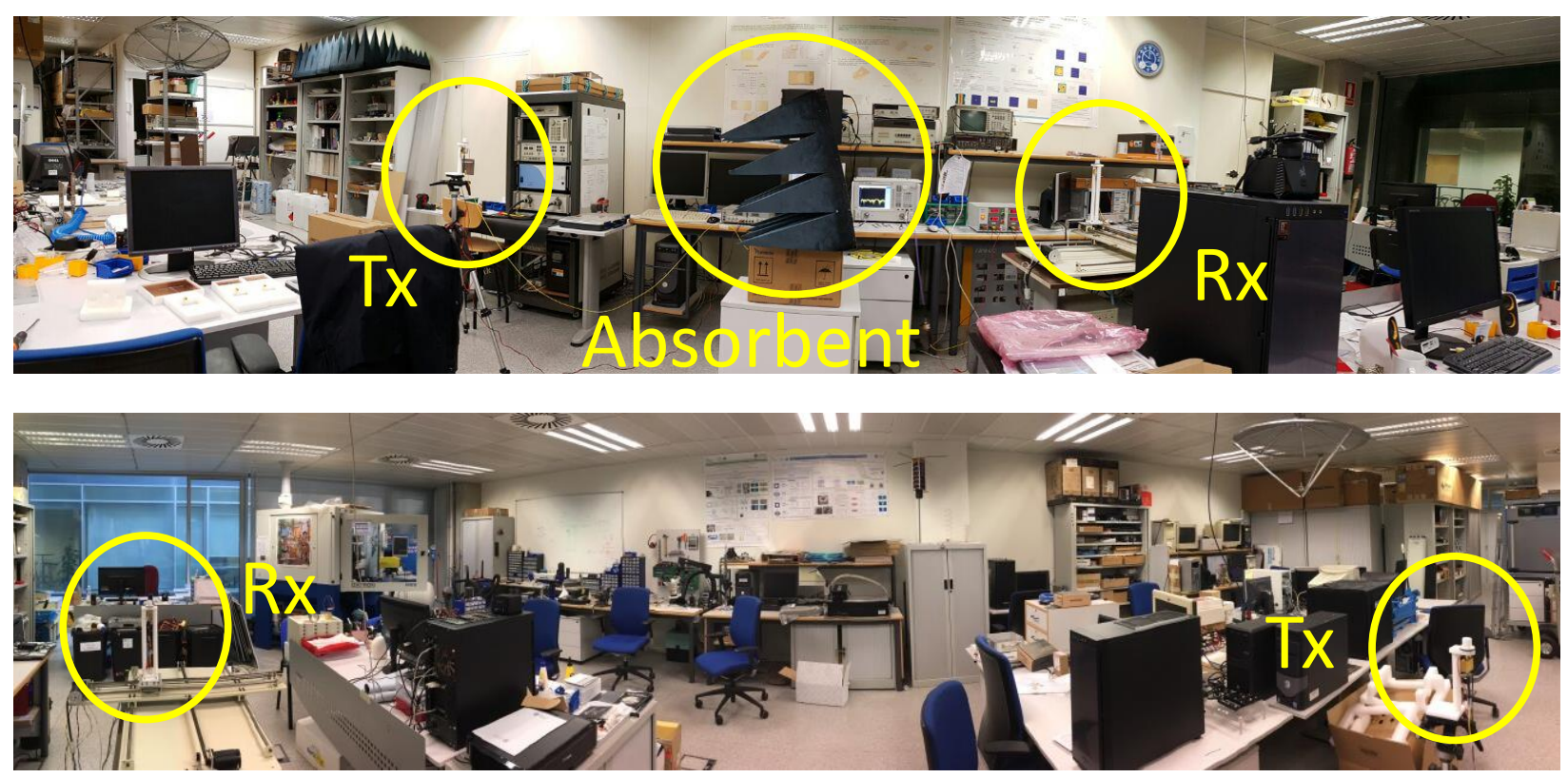

Fig. 2: Laboratory setup for the measurements. (Up) front view, where the transmitting antenna is on the left and the receiving antenna on the right, in the middle of the photograph the absorbent material used in a specific measurement configuration is shown. (Down) back view, where the transmitting antenna is on the right and the receiving antenna on the left.

TABLE II: Scenarios Characteristics

\begin{tabular}{c|c|c|c}
\hline \hline Scenario & Tx-Rx distance $(\mathrm{m})$ & Polarization $(\mathrm{Tx}, \mathrm{Rx})$ & Propagation \\
\hline 1 & $3.29 \mathrm{~m}$ & $(\mathrm{H}, \mathrm{V})$ & $\mathrm{LoS}$ \\
2 & $3.29 \mathrm{~m}$ & $(\mathrm{~V}, \mathrm{~V})$ & $\mathrm{LoS}$ \\
3 & $3.29 \mathrm{~m}$ & $(\mathrm{~V}, \mathrm{~V})$ & $\mathrm{nLoS}$ \\
4 & $2.77 \mathrm{~m}$ & $(\mathrm{~V}, \mathrm{~V})$ & $\mathrm{LoS}$ \\
\hline
\end{tabular}

4 this was about $25.2 \mathrm{~h}$. To guarantee stationary channel conditions, which are affected by the presence of people, the measurements were conducted during weekends.

\section{Results}

\section{A. First-Order Statistics}

As hinted in Section III, the amount of collected data is indeed immense. To keep the analyses manageable for investigation purposes, the frequencies were chosen at the extreme ends and at the middle of the spectrum band, namely, $55 \mathrm{GHz}$ (1st bin), $60.0048828 \mathrm{GHz}$ (1025th bin), 
denoted as $60 \mathrm{GHz}$, and $65 \mathrm{GHz}$ (2048th bin). All four scenarios, as described in Section III, have been explored. In the tests carried out in this paper, the number of collected samples for Scenarios 1, 2 and 3 has been 1225 and for Scenario 4 has been 2500.

For the analysis conducted here, and for each one of the frequencies and for all four scenarios, the parameters of the target fading distributions (i.e., Rayleigh, Rice, Nakagami- $m, \alpha-\mu, \kappa-\mu, \eta-\mu$, and $\alpha-\eta-\kappa-\mu)$ have been estimated. Several built-in functions are available within MatLab that can be used for parameter estimation purposes. For the PDFs case, the Matlab least-square-error based function has been chosen, namely lscurvefit. For fairness, the same method is utilized for all distributions with the same starting point. More details on lsqcurvefit function can be found at [38]. Having the parameters estimated, then the NMSE, the KS as well as the AIC metrics are found.

Table III shows the estimated parameters employing the nonlinear least square method, the values of NMSE, in logarithmic scale, the KS statistic $D$, the corresponding $p$-value and the AIC statistic for each target distribution. Bold-faced numbers highlight the best-fitting result in each performance metric. From Table III, it can be seen that the NMSE, KS, and AIC criteria do not necessarily agree with each other. Whereas through the first criterion for 12 out of 12 tests the best-fittings have been achieved by the generalized fading distributions, through the second one these figures are 11 out of 12. And for the third metric this result are 4 out of 12 . On the other hand, the effect of the polarization combination on the short-term fading distribution can be inferred from the estimated parameters in Scenario 1 and Scenario 2 with LoS condition corresponding to the same Tx and Rx antenna positions and polarization combinations $\mathrm{HV}$ and VV, respectively, as it is shown in Table II. The estimated $\kappa$ parameters of the Rice distribution for the frequencies of 55, 60 and $65 \mathrm{GHz}$ are $0.37,0.22$ and 0.71 for $\mathrm{HV}$ and 2.16, 3.45 and 1.92 for VV. Using HV combination the LoS contribution substantially decreases with a significantly lower level given by the cross-polarization component of both Tx and Rx antennas. Nevertheless, in case of HV the diffuse component does not decrease as the LoS dominant component diminishes. This is due to the fact that the reflected and scattered components suffer polarization changes in the process of reflection and scattering and thus the overall diffuse component in $\mathrm{HV}$ is not significantly lower than in VV. Therefore, mathematically the $\kappa$ parameter is expected to be smaller in HV than in VV. Particularly, in the case of HV polarization for the Scenario 1, the estimated values of the $\kappa$ parameter of the Rice distribution are very close to 0 and consequently the short-term fading distribution can be approximated by a Rayleigh 
TABLE III: (a) PDF Fitting results for Scenario 1 and Scenario 2.

\begin{tabular}{|c|c|c|c|c|c|c|c|c|c|c|c|c|c|}
\hline \multirow{2}{*}{ Scenarios } & \multirow{2}{*}{ Frequency } & \multirow{2}{*}{ Distribution } & \multirow{2}{*}{$\hat{\alpha}$} & \multirow{2}{*}{$\hat{\eta}$} & \multirow{2}{*}{$\hat{\kappa}$} & \multirow{2}{*}{$\hat{\mu}$} & \multirow{2}{*}{$\hat{p}$} & \multirow{2}{*}{$\hat{q}$} & \multirow{2}{*}{$\hat{\hat{r}}$} & \multirow{2}{*}{ NMSE [dB] } & \multicolumn{2}{|c|}{ KS test } & \multirow{2}{*}{ AIC } \\
\hline & & & & & & & & & & & $D$ & $p$-value & \\
\hline \multirow{21}{*}{ Scenario 1} & \multirow{7}{*}{$55 \mathrm{GHz}$} & Rayleigh & - & - & - & - & - & - & 1.01 & -17.46 & 0.02 & 0.70 & -284.25 \\
\hline & & Rice & - & - & 0.37 & - & - & - & 0.99 & -17.57 & 0.02 & 0.66 & -283.45 \\
\hline & & Nakagami- $m$ & - & - & - & 1.03 & - & - & $\approx 1$ & -17.54 & 0.02 & 0.58 & -283.12 \\
\hline & & $\alpha-\mu$ & 2.21 & - & - & 0.89 & - & - & 1.01 & -17.59 & 0.02 & 0.69 & -281.71 \\
\hline & & $\kappa-\mu$ & - & - & 0.43 & 0.99 & - & - & 0.99 & -17.57 & 0.02 & 0.69 & -281.47 \\
\hline & & $\eta-\mu$ & - & $\approx 1$ & - & 1.03 & - & - & $\approx 1$ & -17.54 & 0.25 & $\approx 0$ & -281.12 \\
\hline & & $\alpha-\eta-\kappa-\mu$ & 3.82 & 0.18 & 0.08 & 0.47 & 1.26 & 0.10 & 1.16 & -17.86 & 0.02 & 0.76 & -276.88 \\
\hline & \multirow{7}{*}{$60 \mathrm{GHz}$} & Rayleigh & - & - & - & - & - & - & 1.01 & -15.92 & 0.02 & 0.65 & -255.75 \\
\hline & & Rice & - & - & 0.22 & - & - & - & 1.01 & -15.94 & 0.02 & 0.60 & -253.94 \\
\hline & & Nakagami- $m$ & - & - & - & 0.96 & - & - & 1.02 & -16.02 & 0.02 & 0.56 & -254.83 \\
\hline & & $\alpha-\mu$ & 2.75 & - & - & 0.61 & - & - & 1.05 & -16.40 & 0.01 & 0.98 & -257.27 \\
\hline & & $\kappa-\mu$ & - & - & 0.84 & 0.85 & - & - & 0.99 & -16.38 & 0.01 & 0.99 & -256.99 \\
\hline & & $\eta-\mu$ & - & $\approx 1$ & - & 0.96 & - & - & 1.02 & -16.02 & 0.26 & $\approx 0$ & -252.83 \\
\hline & & $\alpha-\eta-\kappa-\mu$ & 2.37 & 1.27 & 0.53 & 0.71 & 1.26 & 1.70 & 1.02 & -16.42 & 0.01 & 0.98 & -249.45 \\
\hline & \multirow{7}{*}{$65 \mathrm{GHz}$} & Rayleigh & - & - & - & - & - & - & 1.02 & -16.46 & 0.03 & 0.17 & -255.08 \\
\hline & & Rice & - & - & 0.71 & - & - & - & 0.99 & -17.34 & 0.02 & 0.79 & -263.32 \\
\hline & & Nakagami- $m$ & - & - & - & 1.11 & - & - & 1.01 & -17.12 & 0.02 & 0.92 & -260.72 \\
\hline & & $\alpha-\mu$ & 2.45 & - & - & 0.82 & - & - & 1.03 & -17.32 & 0.02 & 0.76 & -261.06 \\
\hline & & $\kappa-\mu$ & - & - & 0.77 & 0.98 & - & - & 0.99 & -17.35 & 0.02 & 0.76 & -261.35 \\
\hline & & $\eta-\mu$ & - & $\approx 1$ & - & 1.11 & - & - & 1.01 & -17.12 & 0.24 & $\approx 0$ & -258.72 \\
\hline & & $\alpha-\eta-\kappa-\mu$ & 4.00 & 5.00 & 1.04 & 0.45 & 0.66 & 1.19 & 1.14 & -17.67 & 0.02 & 0.87 & -257.08 \\
\hline \multirow{21}{*}{ Scenario 2} & \multirow{7}{*}{$55 \mathrm{GHz}$} & Rayleigh & - & - & - & - & - & - & 1.09 & -8.82 & 0.15 & $\approx 0$ & -135.25 \\
\hline & & Rice & - & - & 2.76 & - & - & - & 1.02 & -14.97 & 0.02 & 0.94 & -204.05 \\
\hline & & Nakagami- $m$ & - & - & - & 2.02 & - & - & 1.04 & -14.36 & 0.03 & 0.33 & -196.98 \\
\hline & & $\alpha-\mu$ & 3.70 & - & - & 0.74 & - & - & 1.08 & -15.13 & 0.01 & 0.96 & -203.89 \\
\hline & & $\kappa-\mu$ & - & - & 2.92 & 0.96 & - & - & 1.02 & -14.97 & 0.01 & 0.95 & -202.05 \\
\hline & & $\eta-\mu$ & - & 0.99 & - & 2.00 & - & - & 1.04 & -14.36 & 0.25 & $\approx 0$ & -194.96 \\
\hline & & $\alpha-\eta-\kappa-\mu$ & 5.00 & 0.65 & 1.68 & 0.50 & 2.90 & 0.33 & 1.13 & -15.27 & 0.02 & 0.92 & -197.47 \\
\hline & \multirow{7}{*}{$60 \mathrm{GHz}$} & Rayleigh & - & - & - & - & - & - & 1.10 & -8.01 & 0.18 & $\approx 0$ & -124.16 \\
\hline & & Rice & - & - & 3.45 & - & - & - & 1.01 & -16.68 & 0.01 & 0.97 & -221.90 \\
\hline & & Nakagami- $m$ & - & - & - & 2.38 & - & - & 1.03 & -15.92 & 0.03 & 0.29 & -213.16 \\
\hline & & $\alpha-\mu$ & 3.44 & - & - & 0.95 & - & - & 1.06 & -16.60 & 0.01 & 0.99 & -218.96 \\
\hline & & $\kappa-\mu$ & - & - & 24.47 & 0.19 & - & - & $\approx 1$ & -16.73 & 0.01 & 0.99 & -220.55 \\
\hline & & $\eta-\mu$ & - & 1.04 & - & 2.27 & - & - & 1.03 & -15.84 & 0.23 & $\approx 0$ & -210.25 \\
\hline & & $\alpha-\eta-\kappa-\mu$ & 3.12 & 0.63 & 1.12 & 0.88 & 1.91 & 2.17 & 1.06 & -16.76 & 0.01 & $\approx 1$ & -212.84 \\
\hline & & Rayleigh & - & - & - & - & - & - & 1.07 & -10.64 & 0.10 & $\approx 0$ & -168.42 \\
\hline & & Rice & - & - & 1.92 & - & - & - & $\approx 1$ & -14.92 & 0.02 & 0.70 & -215.62 \\
\hline & & Nakagami- $m$ & - & - & - & 1.63 & - & - & 1.04 & -14.34 & 0.03 & 0.36 & -208.95 \\
\hline & $65 \mathrm{GHz}$ & $\alpha-\mu$ & 3.14 & - & - & 0.79 & - & - & 1.07 & -14.78 & 0.02 & 0.63 & -212.04 \\
\hline & & $\kappa-\mu$ & - & - & 2.66 & 0.83 & - & - & $\approx 1$ & -14.94 & 0.02 & 0.55 & -213.86 \\
\hline & & $\eta-\mu$ & - & $\approx 1$ & - & 1.63 & - & - & 1.04 & -14.34 & 0.25 & $\approx 0$ & -206.95 \\
\hline & & $\alpha-\eta-\kappa-\mu$ & 3.48 & 0.10 & 1.08 & 0.55 & 0.57 & 3.31 & 1.09 & -15.04 & 0.02 & 0.71 & -207.00 \\
\hline
\end{tabular}


TABLE III: (b) PDF Fitting results for Scenario 3 and Scenario 4.

\begin{tabular}{|c|c|c|c|c|c|c|c|c|c|c|c|c|c|}
\hline \multirow{2}{*}{ Scenarios } & \multirow{2}{*}{ Frequency } & \multirow{2}{*}{ Distribution } & \multirow{2}{*}{$\hat{\alpha}$} & \multirow{2}{*}{$\hat{\eta}$} & \multirow{2}{*}{$\hat{\kappa}$} & \multirow{2}{*}{$\hat{\mu}$} & \multirow{2}{*}{$\hat{p}$} & \multirow{2}{*}{$\hat{q}$} & \multirow{2}{*}{$\hat{\hat{r}}$} & \multirow{2}{*}{ NMSE [dB] } & \multicolumn{2}{|c|}{ KS test } & \multirow{2}{*}{ AIC } \\
\hline & & & & & & & & & & & $D$ & $p$-value & \\
\hline \multirow{21}{*}{ Scenario 3} & \multirow{7}{*}{$55 \mathrm{GHz}$} & Rayleigh & - & - & - & - & - & - & 1.05 & -13.64 & 0.05 & $\approx 0$ & -215.78 \\
\hline & & Rice & - & - & 1.29 & - & - & - & $\approx 1$ & -17.13 & 0.02 & 0.83 & -254.01 \\
\hline & & Nakagami- $m$ & - & - & - & 1.32 & - & - & 1.04 & -15.95 & 0.03 & 0.19 & -240.45 \\
\hline & & $\alpha-\mu$ & 3.46 & - & - & 0.57 & - & - & 1.09 & -17.19 & 0.02 & 0.77 & -252.67 \\
\hline & & $\kappa-\mu$ & - & - & 2.04 & 0.81 & - & - & $\approx 1$ & -17.31 & 0.02 & 0.94 & -254.10 \\
\hline & & $\eta-\mu$ & - & $\approx 1$ & - & 1.32 & - & - & 1.04 & -15.95 & 0.25 & $\approx 0$ & -238.45 \\
\hline & & $\alpha-\eta-\kappa-\mu$ & 3.48 & 0.10 & 1.23 & 0.50 & 0.54 & 1.88 & 1.09 & -17.41 & 0.02 & 0.81 & -247.26 \\
\hline & \multirow{7}{*}{$60 \mathrm{GHz}$} & Rayleigh & - & - & - & - & - & - & 1.08 & -8.57 & 0.14 & $\approx 0$ & -138.24 \\
\hline & & Rice & - & - & 2.91 & - & - & - & $\approx 1$ & -16.14 & 0.01 & 0.99 & -223.41 \\
\hline & & Nakagami- $m$ & - & - & - & 2.13 & - & - & 1.03 & -16.02 & 0.02 & 0.44 & -222.03 \\
\hline & & $\alpha-\mu$ & 2.70 & - & - & 1.27 & - & - & 1.05 & -16.21 & 0.02 & 0.88 & -222.13 \\
\hline & & $\kappa-\mu$ & - & - & 1.19 & 1.57 & - & - & 1.01 & -16.19 & 0.02 & 0.89 & -222.00 \\
\hline & & $\eta-\mu$ & - & $\approx 1$ & - & 2.13 & - & - & 1.03 & -16.02 & 0.24 & $\approx 0$ & -220.03 \\
\hline & & $\alpha-\eta-\kappa-\mu$ & 3.08 & 0.29 & 5 & 0.52 & 1.59 & 1.07 & 1.05 & -16.43 & 0.01 & 0.99 & -216.67 \\
\hline & \multirow{7}{*}{$65 \mathrm{GHz}$} & Rayleigh & - & - & - & - & - & - & 1.03 & -15.90 & 0.03 & 0.39 & -251.46 \\
\hline & & Rice & - & - & 0.46 & - & - & - & 1.01 & -16.10 & 0.02 & 0.65 & -251.69 \\
\hline & & Nakagami- $m$ & - & - & - & 1.01 & - & - & 1.03 & -15.90 & 0.03 & 0.42 & -249.48 \\
\hline & & $\alpha-\mu$ & 2.97 & - & - & 0.57 & - & - & 1.07 & -16.51 & 0.01 & 0.96 & -254.47 \\
\hline & & $\kappa-\mu$ & - & - & 1.05 & 0.84 & - & - & $\approx 1$ & -16.47 & 0.01 & 0.99 & -254.04 \\
\hline & & $\eta-\mu$ & - & 1 & - & 1.01 & - & - & 1.03 & -15.90 & 0.26 & $\approx 0$ & -247.48 \\
\hline & & $\alpha-\eta-\kappa-\mu$ & 2.76 & 1.43 & 0.38 & 0.61 & 3.60 & 3.95 & 1.06 & -16.53 & 0.01 & 0.96 & -246.69 \\
\hline \multirow{21}{*}{ Scenario 4} & \multirow{7}{*}{$55 \mathrm{GHz}$} & Rayleigh & - & - & - & - & - & - & 1.10 & -6.61 & 0.22 & $\approx 0$ & -100.27 \\
\hline & & Rice & - & - & 4.94 & - & - & - & $\approx 1$ & -21.11 & 0.01 & 0.93 & -265.30 \\
\hline & & Nakagami- $m$ & - & - & - & 3.13 & - & - & 1.02 & -19.83 & 0.03 & 0.07 & -250.55 \\
\hline & & $\alpha-\mu$ & 3.16 & - & - & 1.39 & - & - & 1.04 & -20.83 & 0.01 & 0.89 & -260.03 \\
\hline & & $\kappa-\mu$ & - & - & 24.43 & 0.25 & - & - & $\approx 1$ & -21.16 & 0.01 & 0.85 & -263.81 \\
\hline & & $\eta-\mu$ & & $\approx 1$ & - & 2.68 & - & - & 1.02 & -18.18 & 0.20 & $\approx 0$ & -229.52 \\
\hline & & $\alpha-\eta-\kappa-\mu$ & 5.00 & 0.95 & 1.43 & 0.71 & 4.69 & 0.43 & 1.10 & -22.12 & 0.01 & 0.99 & -266.92 \\
\hline & \multirow{7}{*}{$60 \mathrm{GHz}$} & Rayleigh & - & - & - & - & - & - & 1.09 & -6.01 & 0.23 & $\approx 0$ & -90.63 \\
\hline & & Rice & - & - & 5.49 & - & - & - & $\approx 1$ & -19.56 & 0.01 & 0.75 & -244.67 \\
\hline & & Nakagami- $m$ & - & - & - & 3.41 & - & - & 1.02 & -19.27 & 0.02 & 0.39 & -241.31 \\
\hline & & $\alpha-\mu$ & 2.78 & - & - & 1.88 & - & - & 1.03 & -19.62 & 0.01 & 0.93 & -243.32 \\
\hline & & $\kappa-\mu$ & - & - & 1.80 & 2.06 & - & - & $\approx 1$ & -19.62 & 0.01 & 0.93 & -243.32 \\
\hline & & $\eta-\mu$ & - & $\approx 1$ & - & 2.95 & - & - & 1.02 & -17.90 & 0.21 & $\approx 0$ & -223.55 \\
\hline & & $\alpha-\eta-\kappa-\mu$ & 5.00 & 0.99 & 1.33 & 0.85 & 0.24 & 5.00 & 1.09 & -19.79 & 0.01 & 0.77 & -237.27 \\
\hline & & Rayleigh & - & - & - & - & - & - & 1.11 & -6.26 & 0.25 & $\approx 0$ & -91.74 \\
\hline & & Rice & - & - & 5.18 & - & - & - & $\approx 1$ & -17.88 & 0.01 & 0.73 & -223.49 \\
\hline & & Nakagami- $m$ & - & - & - & 3.25 & - & - & 1.02 & -17.45 & 0.02 & 0.44 & -218.56 \\
\hline & $65 \mathrm{GHz}$ & $\alpha-\mu$ & 3.08 & - & - & 1.51 & - & - & 1.04 & -17.89 & 0.01 & 0.81 & -221.58 \\
\hline & & $\kappa-\mu$ & - & - & 3.53 & 1.32 & - & - & 1.01 & -17.89 & 0.01 & 0.82 & -221.53 \\
\hline & & $\eta-\mu$ & - & 1.04 & - & 2.75 & - & - & 1.03 & -16.30 & 0.21 & $\approx 0$ & -203.24 \\
\hline & & $\alpha-\eta-\kappa-\mu$ & 5.00 & 0.88 & 2.16 & 0.77 & 0.19 & 5.00 & 1.09 & -18.10 & 0.01 & 0.76 & -216.04 \\
\hline
\end{tabular}


distribution where the dominant component is negligible compared to the diffuse component.

As expected, considering the NMSE and the KS criteria, the more general distributions perform better than the simpler ones, since they have more parameters and thus are more flexible. On the other hand, when considering the AIC metric, we find that in most cases the conventional distributions obtained the best compromise solution. As already mentioned and as well known, the AIC penalizes the distributions with greater number of parameters. However, as can be verified by the results, there are some situations (4 of 12) in which the more general distributions provided an adjustment gain that justify the increase of complexity. It is also worth mentioning that if another information criterion were selected (e.g., Bayesian, Deviance, Focus, Watanabe-Akaike, etc.) other results are found.

To visually illustrate the fitting process, Fig. 3 shows theoretical PDFs plotted alongside the empirical one for Scenario 4 and frequency of $65 \mathrm{GHz}$ as a function of normalized envelope $\rho=R / \sqrt[\alpha]{\mathbb{E}\left(R^{\alpha}\right)}$ in logarithmic units. By visual inspection, it is possible to verify that, with the exception of the Rayleigh case, all the other distributions match rather well the empirical density. In fact, in this particular case, through the objective statistical measure, the following distributions $\alpha-\eta-\kappa-\mu, \alpha-\eta$ and Rice are selected as the ones yielding the best-fitting considering the NMSE, the KS and the AIC metrics, respectively.

The second fitting analysis presented here concerns the lower tail adjustment to the empirical CDFs. This analysis is certainly paramount in modern wireless communication systems, in which equipment is increasingly led to work with very low signal-to-noise ratios. The aim is to find situations in which the metrics, though objectively yielding excellent fitting performance, are far from representing the true picture. For this purpose, Scenario 3 at $60 \mathrm{GHz}$ and Scenario 4 at $65 \mathrm{GHz}$ have been picked. As can be seen in Fig. 4, in the first case, the $\alpha-\eta-\kappa-\mu$ distribution was found to give the best values in the NMSE and KS tests. In the second case, $\alpha-\eta-\kappa-\mu$ and $\alpha-\mu$ have achieved the best-fitting in NMSE and KS tests, respectively. Nonetheless, observe in Fig. 4(a) and Fig. 4(b) how the tails of both distributions fail to follow the true curve altogether. Because of its indisputable flexibility, the $\alpha-\eta-\kappa-\mu$ distribution has been chosen to manually fit the true distribution. The parameters and the curves can be seen in these same Fig. 4(a) and Fig. 4(b). It is noteworthy that the same approximate curve can be obtained for other set of parameters. For instance, the curve of Fig. 4(a) can also be obtained with the following parameters: $\hat{\alpha}=0.99$, $\hat{\eta}=1, \hat{\kappa}=150, \hat{\mu}=0.07, \hat{p}=1.14, \hat{q}=0.998, \hat{r}=29.117 \times 10^{-5}, \mathrm{NMSE}=-12.51 \mathrm{~dB}$, $D=0.0884$, and $p$-value $\approx 0$. Note how well the $\alpha-\eta-\kappa-\mu$ lower tail-fitting distribution tends to 


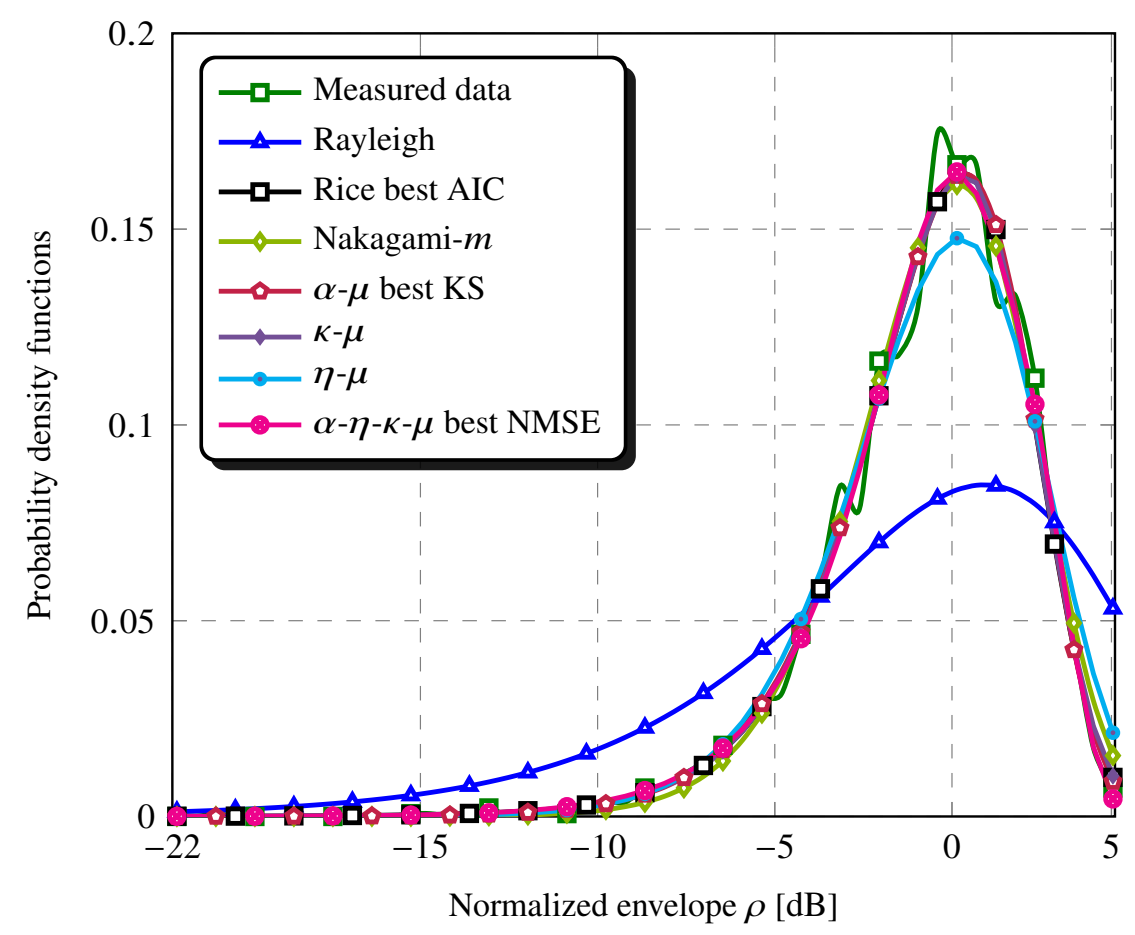

Fig. 3: Estimated vs theoretical PDFs for Scenario 4 at $65 \mathrm{GHz}$.

follow the true curves. In addition, in Scenario 3, the modified KS statistics were 1.4885 and 0.2215 for $\alpha-\eta-\kappa-\mu$ (the best NMSE and KS) and $\alpha-\eta-\kappa-\mu$ lower tail-fitting, respectively. Then, the $\alpha-\eta-\kappa-\mu$ density achieved the best modified KS values in both cases. In the same way, the modified KS statistics were 1.6891, 2.2943 and 0.1688 for $\alpha-\eta-\kappa-\mu$ (the best NMSE), $\alpha-\mu$ (the best KS) and $\alpha-\eta-\kappa-\mu$ lower tail-fitting, respectively.

A general and important conclusion is due. There is not a single combination of physical phenomena that will categorically describe the behavior of the communication channel. Different combinations of physical phenomena, such as non-linearity of the medium, multipath clustering, dominant signal power, among others may result in similar modeling. Take, for instance, Nakagami- $m$ and Rice. In no premises of any physical model for the Nakagami- $m$ distribution a dominant component appears. Only multipath clustering is considered. In this sense, Nakagami$m$ cannot be used for environments within which no dominant components exist. However, the clustering effect emulates that of a dominant component, and Nakagami- $m$ has been successfully applied to both nLoS and LoS situations. The same happens with Weibull and Rice. And this interchangeability of effects, at least in a approximate way, as the case of Rice and Nakagami- $m$, 

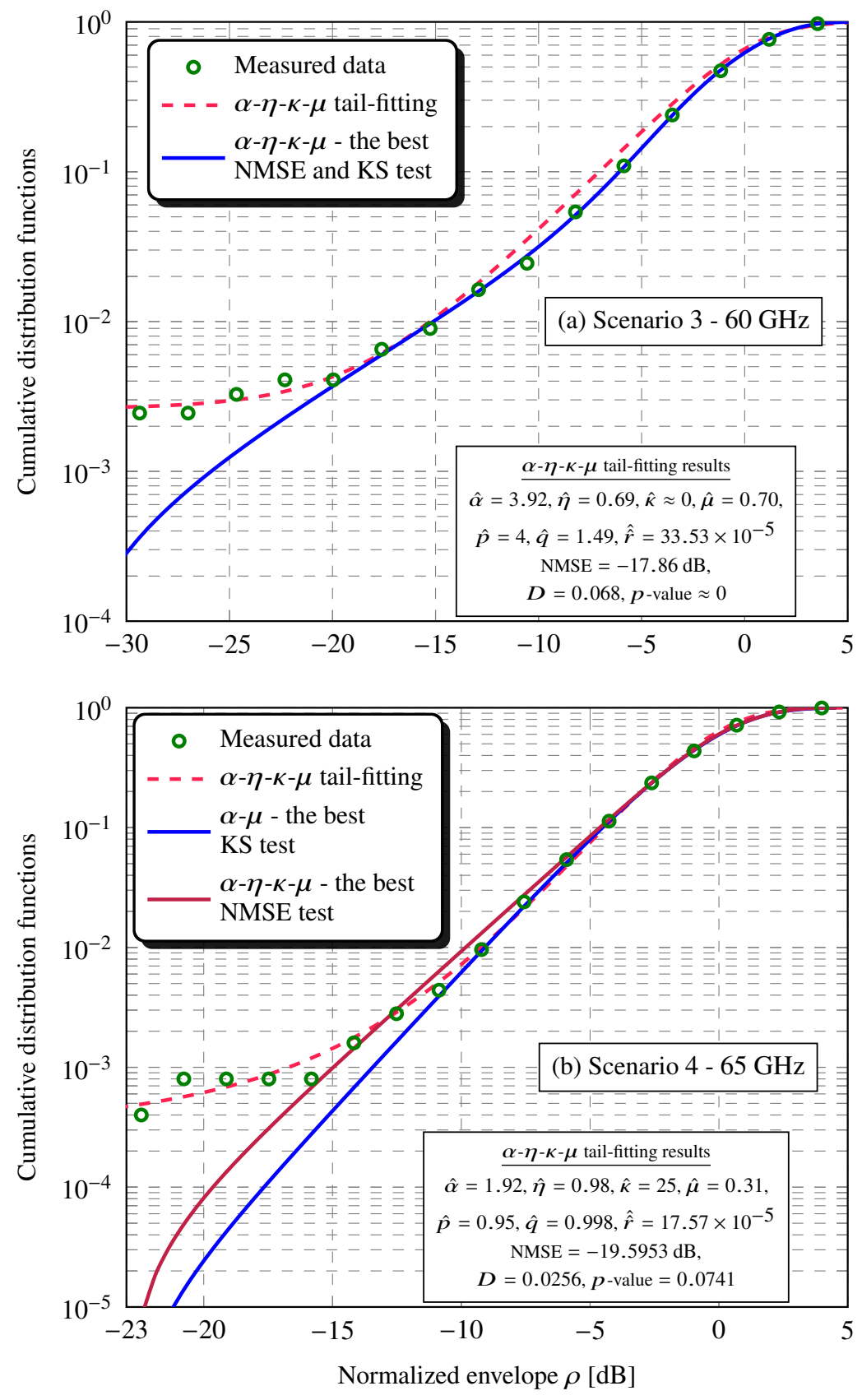

Fig. 4: CDF tail-fitting approach for Scenarios 3 (a) and 4 (b).

is easily and more potentially provided by the $\alpha-\eta-\kappa-\mu$ fading model. In general, the environments are rather complex and there is no single or simple solution either in physical or mathematical terms in channel modeling. 


\section{B. Second-Order Statistics}

In this subsection, the adherence of the theoretical $\mathrm{LCR}_{\mathrm{f}}$ curves, given by (10)-(15) and (17), to the empirical $\mathrm{LCR}_{\mathrm{f}}$ ones in all four scenarios described in Section III is assessed. Again, due to the large amount of collected data and without loss of generality, the position chosen to be evaluated in this analysis for all scenarios has been the $17 \times 17$, located near the center of the URA. For all four scenarios, the parameters of the target $\mathrm{LCR}_{\mathrm{f}}$ fading models (i.e., Rayleigh, Rice, Nakagami- $m, \alpha-\mu, \kappa-\mu, \eta-\mu$, and $\alpha-\eta-\kappa-\mu)$ have also been estimated with the lscurvefit function. Then the NMSE and AIC are calculated aiming to find the best fit for each of the four evaluated scenarios.

Table IV summarizes the parameter estimates and the values of NMSE in logarithmic scale and AIC. From Table IV, it can be seen that in all four evaluated scenarios the best-fitting performance has been achieved by the more general fading models. If we consider the NMSE metric in the four analyzed scenarios, the best performance have been achieved by $\alpha-\eta-\kappa-\mu$. Considering the AIC metric, for Scenarios 1, 2 and 4 the best results have also been obtained by the $\alpha-\eta-\kappa-\mu$. However, in Scenario 3, despite providing a better fit considering the NMSE metric, the $\alpha-\eta-\kappa-\mu$ distribution did not obtain the best performance in the AIC test. This is justifiable, since the AIC test penalizes distributions with more parameters, which is the case of the $\alpha-\eta-\kappa-\mu$ (7 parameters). In fact, in Scenario 3, the distribution that provided the best result in the AIC test has been the $\kappa-\mu$ (2 parameters), being in this way, for Scenario 3 , the best distribution considering a compromise between fit and complexity.

A typical plot of the variation of the amplitude as a function of the frequency is shown in Fig. 5. In the same way, the corresponding autocorrelation function is given in Fig. 6.

To illustrate the $\mathrm{LCR}_{\mathrm{f}}$ fitting process, Fig. 7 shows the theoretical $\mathrm{LCR}_{\mathrm{f}}$ curves plotted alongside the empirical one for all four scenarios as a function of the normalized enveloped $\rho$ in logarithmic units. By visual inspection, it is possible to verify that, for Scenario 1, with the exceptions of Rayleigh and Rice distributions, all the other distributions match rather well the empirical $\mathrm{LCR}_{\mathrm{f}}$. However, if we verify only the extremely lower tail portion of the plots, i.e., for substantially small $\rho$, the $\alpha-\eta-\kappa-\mu$ and $\eta-\mu$ yield a better adherence. In fact, the bestfitting considering the NMSE and AIC metrics is found for $\alpha-\eta-\kappa-\mu$ distribution in this case. For Scenario 2, with exception of Rayleigh, all the other fading models achieved a reasonable fitting. Analyzing the lower tail, the best visual adjustment has been obtained by $\kappa-\mu$. In this 
TABLE IV: $\mathrm{LCR}_{\mathrm{f}}$ fitting results.

\begin{tabular}{|c|c|c|c|c|c|c|c|c|c|c|c|}
\hline Scenarios & Distribution & $\hat{\alpha}$ & $\hat{\eta}$ & $\hat{\kappa}$ & $\hat{\mu}$ & $\hat{p}$ & $\hat{q}$ & $\hat{d}$ & $\hat{\tilde{\psi}}\left[\times 10^{3}\right]$ & NMSE[dB] & AIC \\
\hline \multirow{7}{*}{ Scenario 1} & Rayleigh & - & - & - & - & - & - & - & -10.41 & -14.58 & 157.75 \\
\hline & Rice & - & - & 0.96 & - & - & - & - & -22.18 & -17.19 & 108.78 \\
\hline & Nakagami- $m$ & - & - & - & 1.22 & - & - & - & -13.72 & -23.67 & -18.13 \\
\hline & $\alpha-\mu$ & 2.03 & - & - & 1.21 & - & - & - & -13.75 & -23.69 & -16.44 \\
\hline & $\kappa-\mu$ & - & - & $\approx 0$ & 1.22 & - & - & - & -13.72 & -23.67 & -16.13 \\
\hline & $\eta-\mu$ & - & 0.19 & - & 0.70 & - & - & - & -12.66 & -31.46 & -168.66 \\
\hline & $\alpha-\eta-\kappa-\mu$ & 1.79 & 0.71 & 0.39 & 1.51 & 0.32 & 21.26 & 0.56 & -18.33 & -32.36 & -176.32 \\
\hline \multirow{7}{*}{ Scenario 2} & Rayleigh & - & - & - & - & - & - & - & -5.68 & -7.47 & 206.81 \\
\hline & Rice & - & - & 2.70 & - & - & - & - & -26.43 & -23.95 & -22.66 \\
\hline & Nakagami-m & - & - & - & 1.94 & - & - & - & -13.36 & -25.43 & -43.45 \\
\hline & $\alpha-\mu$ & 1.94 & - & - & 2.01 & - & - & - & -13.30 & -25.54 & -42.99 \\
\hline & $\kappa-\mu$ & - & - & 0.90 & 1.59 & - & - & - & -21.34 & -29.04 & -97.17 \\
\hline & $\eta-\mu$ & - & 0.99 & - & 0.96 & - & - & - & -13.11 & -25.34 & -40.21 \\
\hline & $\alpha-\eta-\kappa-\mu$ & 2.17 & 9.43 & 0.36 & 1.28 & 2.05 & $\approx 0$ & 4.20 & -7.27 & -31.60 & -118.06 \\
\hline \multirow{7}{*}{ Scenario 3} & Rayleigh & - & - & - & - & - & - & - & -6.32 & -9.96 & 243.52 \\
\hline & Rice & - & - & 1.93 & - & - & - & - & -22.25 & -26.53 & -170.29 \\
\hline & Nakagami- $m$ & - & - & - & 1.55 & - & - & - & -11.31 & -22.90 & -79.39 \\
\hline & $\alpha-\mu$ & 1.86 & - & - & 1.66 & - & - & - & -11.20 & -23.24 & -85.95 \\
\hline & $\kappa-\mu$ & - & - & 1.16 & 1.22 & - & - & - & -19.85 & -32.36 & -314.73 \\
\hline & $\eta-\mu$ & - & $\approx 1$ & - & 0.77 & - & - & - & -11.32 & -22.90 & -77.38 \\
\hline & $\alpha-\eta-\kappa-\mu$ & 1.87 & 0.74 & 4.24 & 1.21 & 0.28 & 16.64 & 0.45 & -47.35 & -32.59 & -310.46 \\
\hline \multirow{7}{*}{ Scenario 4} & Rayleigh & - & - & - & - & - & - & - & -3.64 & -5.85 & 183.34 \\
\hline & Rice & - & - & 3.62 & - & - & - & - & -21.74 & -22.07 & -5.08 \\
\hline & Nakagami- $m$ & - & - & - & 2.42 & - & - & - & -10.99 & -26.44 & -56.42 \\
\hline & $\alpha-\mu$ & 1.87 & - & - & 2.64 & - & - & - & -10.89 & -27.02 & -61.26 \\
\hline & $\kappa-\mu$ & - & - & 0.44 & 2.25 & - & - & - & -14.84 & -26.94 & -60.28 \\
\hline & $\eta-\mu$ & & 0.99 & - & 1.21 & - & - & - & -10.99 & -26.44 & -54.42 \\
\hline & $\alpha-\eta-\kappa-\mu$ & 1.81 & 3.20 & 8.86 & 0.39 & 0.83 & 0.12 & 0.56 & -20.49 & -29.15 & -76.22 \\
\hline
\end{tabular}

case, the best-fitting considering the NMSE and AIC metrics has been obtained also by the $\alpha-\eta-\kappa-\mu$ model followed by $\kappa-\mu$. For Scenario 3, considering $\rho>-18 \mathrm{~dB}$, the model yielding the best visual fitting is the Rician one. On the other hand, at the lower tail the $\alpha-\eta-\kappa-\mu$ and $\kappa-\mu$ models could match the experimental $\mathrm{LCR}_{\mathrm{f}}$. It is important to emphasize that in this scenario the system operated in nLoS condition. In this situation the best-fitting considering the NMSE has been achieved by the $\alpha-\eta-\kappa-\mu$ distribution and the best fit considering the AIC has been achieved by the $\kappa-\mu$ model. Finally, for Scenario 4, considering $\rho>-5 \mathrm{~dB}$, with exception of Rayleigh all other tested models fit perfectly. For $-15<\rho<10$, with exception of Rayleigh and Rice all other models match rather well the experimental curve. At the very low tail, i.e. $\rho<-15$, the empirical curve tends to match those from $\alpha-\mu, \kappa-\mu$ and $\eta-\mu$ models. 


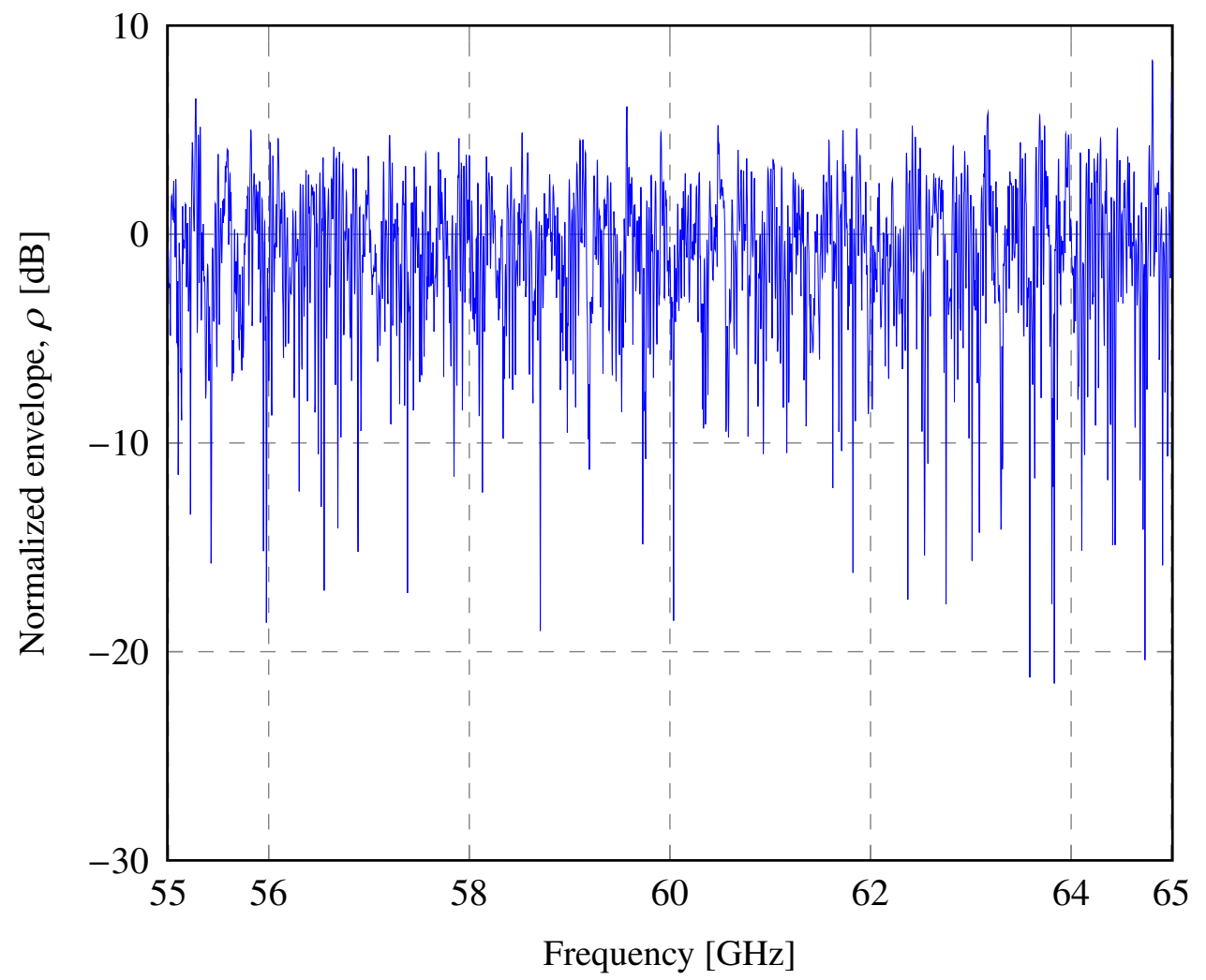

Fig. 5: Amplitude as a function of the frequency (Scenario 2).

\section{Conclusions}

A thorough field measurement campaign in the mm-wave band has been conducted in an indoor environment with an aim at characterizing the first and second order statistics of the short-term fading signal. The frequencies ranged from $55 \mathrm{GHz}$ to $65 \mathrm{GHz}$, and the propagation conditions included LoS and $\mathrm{nLoS}$, with combinations of horizontal and vertical polarizations at both transmitter and receiver. The motivation to use more general fading models, including the $\alpha-\eta-\kappa-\mu$ one, is rather straightforward. The short-term fading has been barely explored in the $60 \mathrm{GHz}$ band. In such a yet unknown scenario, we wanted to investigate whether there would be situations for which the available fading models would not yield a reasonable fit. In this case, the use of the $\alpha-\eta-\kappa-\mu$ could fill this gap. The response to this question has indeed been given in the paper. Indeed, there are such situations, although rather rare. It can be said that the available distributions, namely $\alpha-\mu, \eta-\mu, \kappa-\mu$, and their particular cases can be applied to most of the situations. But, in some rare cases, the $\alpha-\eta-\kappa-\mu$ would yield a better fitting performance. 


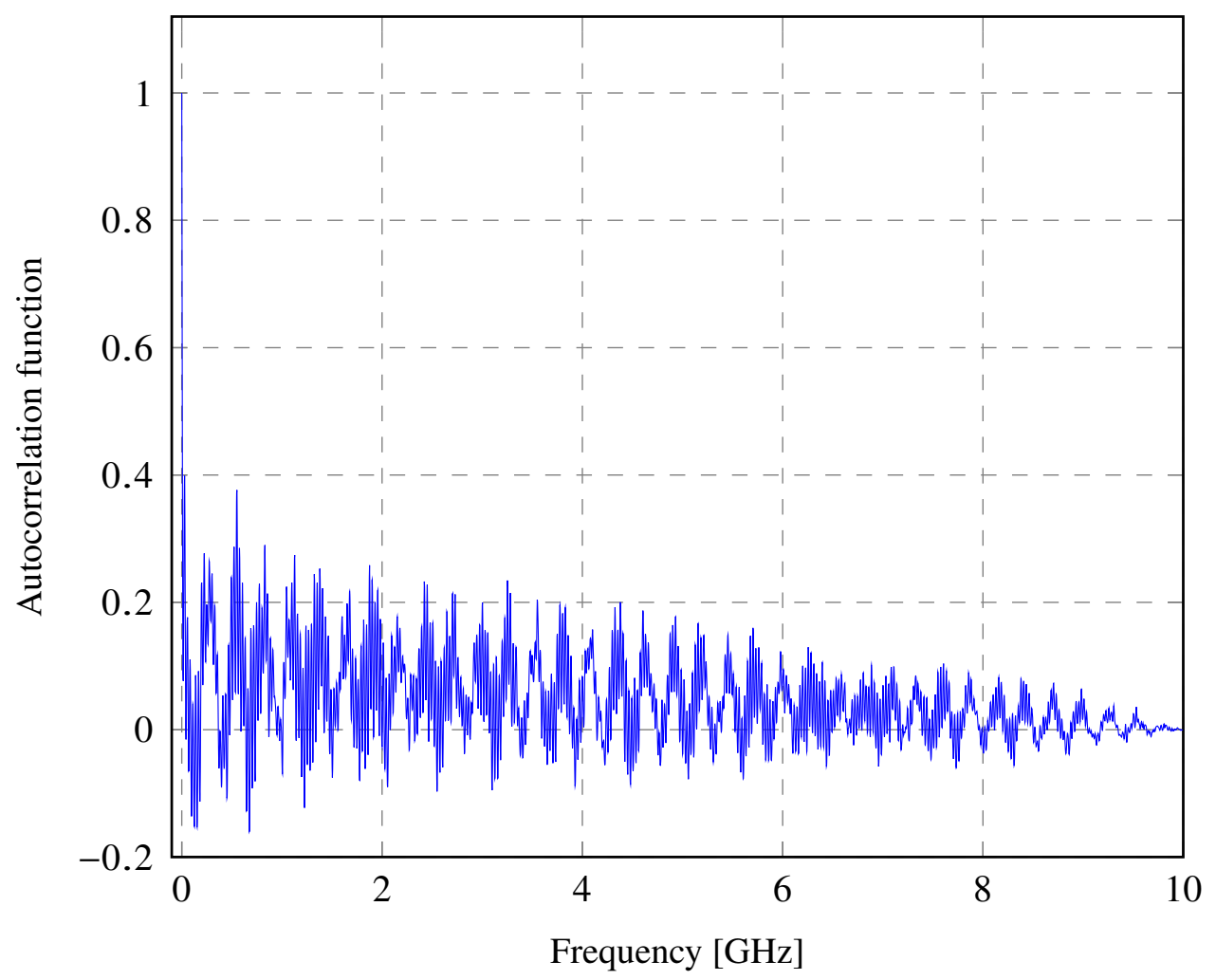

Fig. 6: Autocorrelation as a function of the frequency (Scenario 2).

A number of $\mathrm{LCR}_{\mathrm{f}}$ expressions of multiparameter fading models have been shown here. First and second order statistics (PDF, CDF and $\mathrm{LCR}_{\mathrm{f}}$ ) of the fading models have been evaluated. The models that best fitted the experimental data were chosen using three figures of merit, namely NMSE, KS statistics and AIC. Analyzing the results of the first order statistical tests, it was possible to verify that the most general distribution, namely $\alpha-\eta-\kappa-\mu$, provided the smallest NMSE estimation error in all 12 scenarios analyzed. Considering the KS method, the more general distributions, namely $\alpha-\mu, \eta-\mu$, and $\kappa-\mu$, yielded the best performance in 11 out of 12 scenarios. Finally, in the AIC metric, the more general fading models performed best in 4 out of 12 scenarios.

For the second-order statistical tests, in all four $\mathrm{LCR}_{\mathrm{f}}$ estimated scenarios, as expected, the $\alpha-\eta-\kappa-\mu$ model also presented the best fit performance considering the NMSE method. Moreover, in 3 out of 4 scenarios, the most generalized distribution also obtained the best result in the AIC test, showing that the use of the most complex distribution is in most cases rewarded by a much better curve fit. 

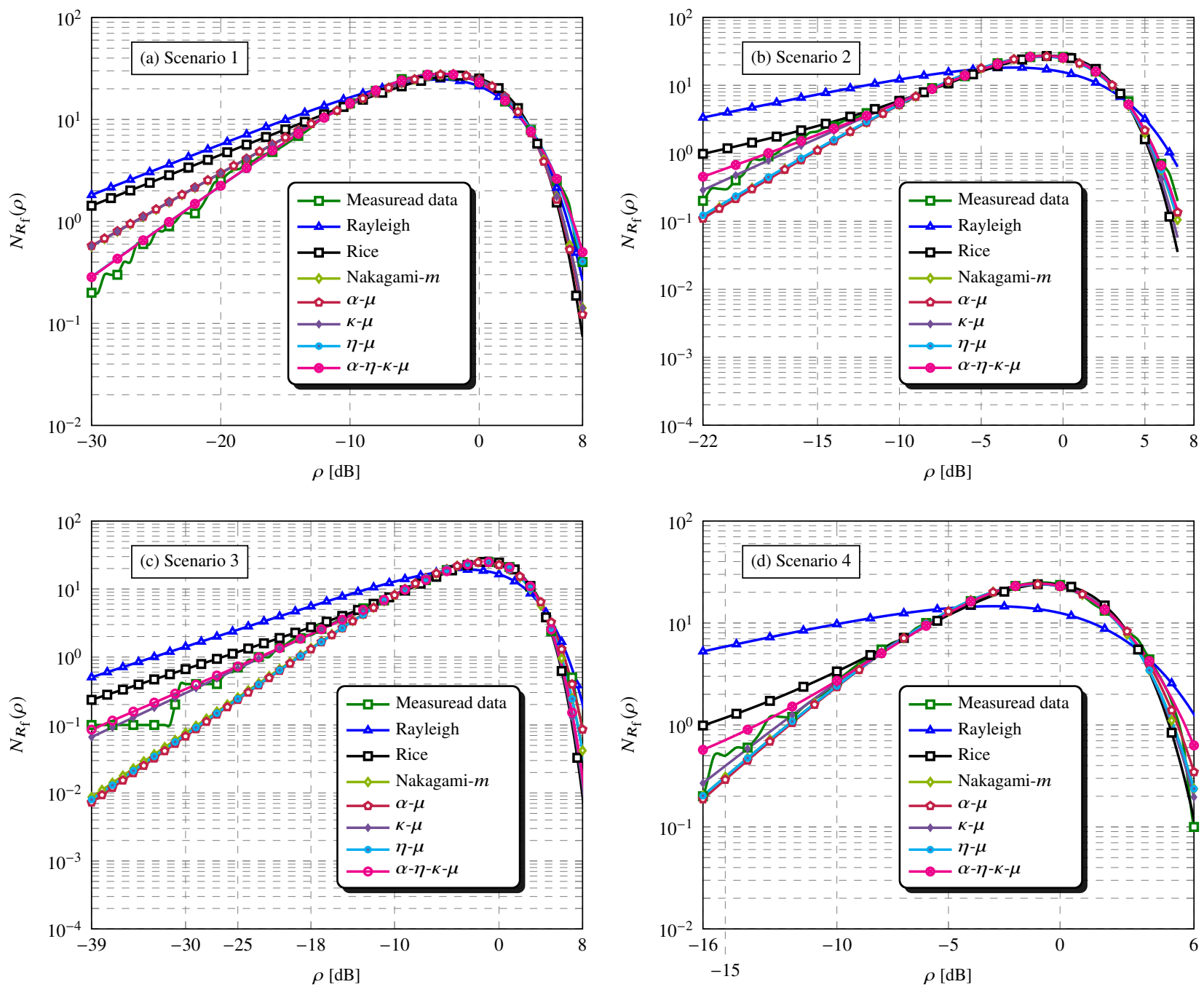

Fig. 7: $\mathrm{LCR}_{\mathrm{f}}$ fitting approach for Scenarios 1 (a), 2 (b), 3 (c) and 4 (d).

Interestingly, it has been observed that in some few situations, the lower tail of the true distribution, obtained with field data, can only be followed by the more general fading model, because of its inherent flexibility. It is noteworthy that the fading models available in the literature fully meet the need for modeling short-term fading in the mm-wave band.

The message of this paper was not to prove beyond doubt that the $\alpha-\eta-\kappa-\mu$ is the fading model to be applied in a millimeter wave scenario. On the contrary, the idea is to show that those fading models already available in the literature, more specifically $\alpha-\mu, \eta-\mu$, and $\kappa-\mu$, suffice. In some occasions, rare ones, apart from $\alpha-\eta-\kappa-\mu$, the fitting performance of the distributions is not as good. Then the $\alpha-\eta-\kappa-\mu$ is the best option. But, of course, this per se, does not justify its 
use in all occasions. As well understood, the $\alpha-\eta-\kappa-\mu$ is mathematically much more intricate than the others. However, due to its multi-parameter feature, the $\alpha-\eta-\kappa-\mu$ distribution provides more flexibility. Obviously, in practice, those general, but simpler, distributions may be the best choice for the vast majority of scenarios considering the trade-off between small error and high complexity.

\section{REFERENCES}

[1] T. S. Rappaport, Y. Xing, G. R. MacCartney, A. F. Molisch, E. Mellios, and J. Zhang, "Overview of millimeter wave communications for fifth-generation (5G) wireless networks - with a focus on propagation models," IEEE Trans. Antennas Propag., vol. 65, no. 12, pp. 6213-6230, Dec. 2017.

[2] J. G. Andrews, T. Bai, M. N. Kulkarni, A. Alkhateeb, A. K. Gupta, and R. W. Heath, "Modeling and analyzing millimeter wave cellular systems," IEEE Trans. Commun., vol. 65, no. 1, pp. 403-430, Jan. 2017.

[3] F. Boccardi, R. W. Heath, A. Lozano, T. L. Marzetta, and P. Popovski, "Five disruptive technology directions for 5G," IEEE Commun. Mag., vol. 52, no. 2, pp. 74-80, Feb. 2014.

[4] T. Zwick, T. J. Beukema, and H. Nam, "Wideband channel sounder with measurements and model for the $60 \mathrm{GHz}$ indoor radio channel," IEEE Trans. Veh. Technol., vol. 54, no. 4, pp. 1266-1277, Jul. 2005.

[5] H. Xu, V. Kukshya, and T. S. Rappaport, "Spatial and temporal characteristics of 60-GHz indoor channels," IEEE J. Sel. Areas Commun., vol. 20, no. 3, pp. 620-630, Apr. 2002.

[6] C. R. Anderson and T. S. Rappaport, "In-building wideband partition loss measurements at 2.5 and 60 GHz," IEEE Trans. Wireless Commun., vol. 3, no. 3, pp. 922-928, May 2004.

[7] P. F. M. Smulders, "Statistical characterization of 60-GHz indoor radio channels," IEEE Trans. Antennas Propag., vol. 57, no. 10 , pp. 2820-2829, Oct. 2009.

[8] H. J. Thomas, R. S. Cole, and G. L. Siqueira, "An experimental study of the propagation of 55 GHz millimeter waves in an urban mobile radio environment," IEEE Trans. Veh. Technol., vol. 43, no. 1, pp. 140-146, Feb. 1994.

[9] M. Kyro, K. Haneda, J. Simola, K. i. Takizawa, H. Hagiwara, and P. Vainikainen, "Statistical channel models for 60 GHz radio propagation in hospital environments," IEEE Trans. Antennas Propag., vol. 60, no. 3, pp. 1569-1577, Mar. 2012.

[10] T. S. Rappaport, G. R. MacCartney, M. K. Samimi, and S. Sun, "Wideband millimeter-wave propagation measurements and channel models for future wireless communication system design,” IEEE Trans. Commun., vol. 63, no. 9, pp. 3029-3056, Sep. 2015.

[11] K. Haneda, J. Jarvelainen, A. Karttunen, M. Kyro, and J. Putkonen, "Indoor short-range radio propagation measurements at 60 and 70 GHz," in The 8th European Conference on Antennas and Propagation (EuCAP 2014), Apr. 2014, pp. 634-638.

[12] S. Hur, S. Baek, B. Kim, Y. Chang, A. F. Molisch, T. S. Rappaport, K. Haneda, and J. Park, "Proposal on millimeter-wave channel modeling for 5g cellular system,” IEEE J. Sel. Topics Signal Process., vol. 10, no. 3, pp. 454-469, Apr. 2016.

[13] M. K. Samimi, G. R. MacCartney, S. Sun, and T. S. Rappaport, "28 GHz millimeter-wave ultrawideband small-scale fading models in wireless channels," in 2016 IEEE 83rd Vehicular Technology Conference (VTC Spring), May 2016, pp. 1-6.

[14] M.-S. Choi, G. Grosskopf, and D. Rohde, "Statistical characteristics of $60 \mathrm{GHz}$ wideband indoor propagation channel," in 2005 IEEE 16th International Symposium on Personal, Indoor and Mobile Radio Communications, vol. 1, Sep. 2005, pp. 599-603.

[15] T. Mavridis, L. Petrillo, J. Sarrazin, A. Benlarbi-Delai, and P. D. Doncker, "Near-body shadowing analysis at 60 GHz," IEEE Trans. Antennas Propag., vol. 63, no. 10, pp. 4505-4511, Oct. 2015. 
[16] J. Reig, M.-T. Martinez-Inglés, L. Rubio, V.-M. Rodrigo-Penarrocha, and J.-M. Molina-García-Pardo, "Fading evaluation in the $60 \mathrm{GHz}$ band in line-of-sight conditions," International Journal of Antennas and Propagation, vol. 2014, p. 12, 2014.

[17] J. Blumenstein, T. Mikulasek, T. Zemen, C. Mecklenbrauker, R. Marsalek, and A. Prokes, "In-vehicle mm-wave channel model and measurement," in 2014 IEEE 80th Vehicular Technology Conference (VTC2014-Fall), Sep. 2014, pp. 1-5.

[18] J. Blumenstein, A. Prokes, A. Chandra, T. Mikulasek, R. Marsalek, T. Zemen, and C. Mecklenbrauker, "In-vehicle channel measurement, characterization, and spatial consistency comparison of 30-11 GHz and 55-65 GHz frequency bands," IEEE Trans. Veh. Technol., vol. 66, no. 5, pp. 3526-3537, May 2017.

[19] M. D. Yacoub, “The $\alpha-\eta-\kappa-\mu$ fading model,” IEEE Trans. Antennas Propag., vol. 64, no. 8, pp. 3597-3610, Aug. 2016.

[20] A. A. dos Anjos, T. R. R. Marins, R. A. A. de Souza, and M. D. Yacoub, "Higher order statistics for the $\alpha-\eta-\kappa-\mu$ fading model," IEEE Trans. Antennas Propag., vol. 66, no. 6, pp. 3002-3016, Jun. 2018.

[21] R. Zhang, Z. Zhong, Y. Zhang, S. Lu, and L. Cai, "Measurement and analytical study of the correlation properties of subchannel fading for noncontiguous carrier aggregation,” IEEE Trans. Veh. Technol., vol. 63, no. 9, pp. 4165-4177, Nov. 2014.

[22] S. Dixit and H. Katiyar, "Performance analysis of OFDM under frequency selective fading in varying power delay profile," in 2016 International Conference on Emerging Trends in Electrical Electronics Sustainable Energy Systems (ICETEESES), Mar. 2016, pp. 213-215.

[23] M. D. Yacoub, J. E. V. Bautista, and L. G. de Rezende Guedes, "On higher order statistics of the Nakagami- $m$ distribution," IEEE Trans. Veh. Technol., vol. 48, no. 3, pp. 790-794, May 1999.

[24] K. Witrisal, Y.-H. Kim, and R. Prasad, "A new method to measure parameters of frequency-selective radio channels using power measurements," IEEE Trans. Commun., vol. 49, no. 10, pp. 1788-1800, Oct. 2001.

[25] A. Abdi, K. Wills, H. A. Barger, M. S. Alouini, and M. Kaveh, "Comparison of the level crossing rate and average fade duration of Rayleigh, Rice and Nakagami fading models with mobile channel data," in 52nd Vehicular Technology Conference Fall 2000., vol. 4, Sep. 2000, pp. 1850-1857.

[26] M. D. Yacoub, Foundations of Mobile Radio Engineering, 1st ed. Boca Raton, FL, USA: CRC Press, Inc., 1993.

[27] — - "The $\alpha-\mu$ distribution: A physical fading model for the Stacy distribution," IEEE Trans. Veh. Technol., vol. 56, no. 1, pp. 27-34, Jan. 2007.

[28] S. L. Cotton and W. G. Scanlon, "Higher-order statistics for $\kappa-\mu$ distribution," Electronics Letters, vol. 43, no. 22 , Oct. 2007.

[29] D. B. da Costa, J. C. S. S. Filho, M. D. Yacoub, and G. Fraidenraich, "Second-order statistics of $\eta-\mu$ fading channels: Theory and applications," IEEE Trans. Veh. Technol., vol. 7, no. 3, pp. 819-824, Mar. 2008.

[30] I. Gradshteyn and I. Ryzhik, Tables of Integrals, Series, and Products. New York: Academic Press, 1980.

[31] P. Chen, T. . Wu, and J. Yang, "A comparative study of model selection criteria for the number of signals," IET Radar, Sonar Navigation, vol. 2, no. 3, pp. 180-188, Jun. 2008.

[32] A. Mariani, A. Giorgetti, and M. Chiani, "Model order selection based on information theoretic criteria: Design of the penalty," IEEE Trans. Signal Process., vol. 63, no. 11, pp. 2779-2789, Jun. 2015.

[33] A. Seghouane, “The Akaike information criterion with parameter uncertainty," in Fourth IEEE Workshop on Sensor Array and Multichannel Processing, 2006., Jul. 2006, pp. 430-434.

[34] J. Ding, V. Tarokh, and Y. Yang, "Model selection techniques: an overview," IEEE Signal Process. Mag., vol. 35, no. 6, pp. 16-34, Nov. 2018.

[35] J. K. Nielsen, M. G. Christensen, and S. H. Jensen, "Bayesian model comparison and the bic for regression models," in 2013 IEEE International Conference on Acoustics, Speech and Signal Processing, May 2013, pp. 6362-6366. 
[36] P. Stoica and Y. Selen, "Model-order selection: a review of information criterion rules," IEEE Signal Process. Mag., vol. 21, no. 4, pp. 36-47, Jul. 2004.

[37] J. M. Romero-Jerez, F. J. Lopez-Martinez, J. F. Paris, and A. J. Goldsmith, “The fluctuating two-ray fading model: Statistical characterization and performance analysis," IEEE Trans. Wireless Commun., vol. 16, no. 7, pp. 4420-4432, Jul. 2017.

[38] MathWorks. [Online]. Available: https://www.mathworks.com/help/optim/ug/lsqcurvefit.html 